\title{
Article
}

\section{The diverse nature and formation paths of slow rotator galaxies in the eagle simulations}

Lagos, Claudia del P, Emsellem, Eric, van de Sande, Jesse, Harborne, Katherine E, Cortese, Luca, Davison, Thomas, Foster, Caroline and Wright, Ruby J

Available at http://clok.uclan.ac.uk/39836/

Lagos, Claudia del P, Emsellem, Eric, van de Sande, Jesse, Harborne, Katherine E, Cortese, Luca, Davison, Thomas, Foster, Caroline and Wright, Ruby J (2022) The diverse nature and formation paths of slow rotator galaxies in the eagle simulations. Monthly Notices of the Royal Astronomical Society, 509 (3). pp. 4372-4391. ISSN 0035-8711

It is advisable to refer to the publisher's version if you intend to cite from the work. http://dx.doi.org/10.1093/mnras/stab3128

For more information about UCLan's research in this area go to

http://www.uclan.ac.uk/researchgroups/ and search for <name of research Group>.

For information about Research generally at UCLan please go to http://www.uclan.ac.uk/research/

All outputs in CLoK are protected by Intellectual Property Rights law, including Copyright law. Copyright, IPR and Moral Rights for the works on this site are retained by the individual authors and/or other copyright owners. Terms and conditions for use of this material are defined in the policies page. 


\title{
The diverse nature and formation paths of slow rotator galaxies in the EAGLE simulations
}

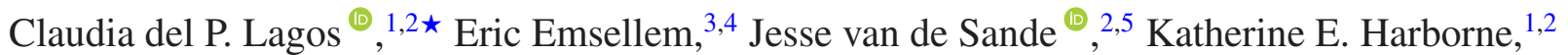 \\ Luca Cortese ${ }^{\circledR}, 1,2$ Thomas Davison ${ }^{\circledR}, 3,6$ Caroline Foster ${ }^{\circledR 2,5}$ and Ruby J. Wright ${ }^{\circledR 1,2}$ \\ ${ }^{1}$ International Centre for Radio Astronomy Research (ICRAR), M468, University of Western Australia, 35 Stirling Hwy, Crawley, WA 6009, Australia \\ ${ }^{2}$ ARC of Excellence for All Sky Astrophysics in 3 Dimensions (ASTRO 3D) \\ ${ }^{3}$ European Southern Observatory, Karl-Schwarzschild-Str. 2, D-85748 Garching, Germany \\ ${ }^{4}$ University of Lyon, ENS de Lyon, CNRS, Centre de Recherche Astrophysique de Lyon, UMR5574, F-69230 Saint-Genis-Laval, France \\ ${ }^{5}$ Sydney Institute for Astronomy, School of Physics, A28, The University of Sydney, NSW 2006, Australia \\ ${ }^{6}$ Jeremiah Horrocks Institute, University of Central Lancashire, Preston PR1 2HE, UK
}

Accepted 2021 October 24. Received 2021 October 8; in original form 2020 December 14

\begin{abstract}
We use a sample of $z=0$ galaxies visually classified as slow rotators (SRs) in the EAGLE hydrodynamical simulations to explore the effect of galaxy mergers on their formation, characterize their intrinsic galaxy properties, and study the connection between quenching and kinematic transformation. SRs that have had major or minor mergers (mass ratios $\geq 0.3$ and $0.1-0.3$, respectively) tend to have a higher triaxiality parameter and ex-situ stellar fractions than those that had exclusively very minor mergers or formed in the absence of mergers ('no-merger' SRs). No-merger SRs are more compact, have lower black hole-to-stellar mass ratios and quenched later than other SRs, leaving imprints on their $z=0$ chemical composition. For the vast majority of SRs we find that quenching, driven by active galactic nuclei feedback, precedes kinematic transformation, except for satellite SRs, in which these processes happen in tandem. However, in $\approx 50$ per cent of these satellites, satellite-satellite mergers are responsible for their SR fate, while environment (i.e. tidal field and interactions with the central) can account for the transformation in the rest. By splitting SRs into kinematic sub-classes, we find that flat SRs prefer major mergers; round SRs prefer minor or very minor mergers; prolate SRs prefer gas-poor mergers. Flat and prolate SRs are more common among satellites hosted by massive haloes $\left(>10^{13.6} \mathrm{M}_{\odot}\right)$ and centrals of high masses $\left(M_{\star}>10^{10.5} \mathrm{M}_{\odot}\right)$. Although EAGLE galaxies display kinematic properties that broadly agree with observations, there are areas of disagreement, such as inverted stellar age and velocity dispersion profiles. We discuss these and how upcoming simulations can solve them.
\end{abstract}

Key words: galaxies: evolution-galaxies: formation-galaxies: kinematics and dynamics-galaxies: structure.

\section{INTRODUCTION}

The advent of integral field spectroscopy (IFS) and large IFS surveys, such as ATLAS ${ }^{3 D}$ (Cappellari et al. 2011), the Sydney-AAO MultiObject Integral-Field Spectrograph (SAMI) Galaxy Survey (Croom et al. 2012; Bryant et al. 2015), the Calar Alto Legacy Integral Field Area Survey (CALIFA; Sánchez et al. 2012), MASSIVE (Ma et al. 2014), and the Mapping Nearby Galaxies at Apache Point Observatory (MaNGA) survey (Bundy et al. 2015), have contributed to significantly expand our understanding of galaxy kinematics and their connection to intrinsic galaxy properties and their environment (e.g. see Cappellari 2016 for a review on kinematics of earlytype galaxies). Among the kinematic parameters that have been most studied in the literature is the stellar spin parameter, $\lambda_{\mathrm{r}}$, first introduced by Emsellem et al. (2007). $\lambda_{\mathrm{r}}$ provides a measurement of how rotationally supported a galaxy is, and strongly correlates with the stellar rotation-to-velocity dispersion ratio (Emsellem et al. 2011; van de Sande et al. 2017b; Harborne et al. 2020b). The study

^E-mail: claudia.lagos@icrar.org of galaxies in the $\lambda_{\mathrm{r}}$-ellipticity $(\epsilon)$ plane led Emsellem et al. (2007, 2011) to coin the terms slow and fast rotators.

IFS surveys have unveiled various correlations between $\lambda_{\mathrm{r}}$ and galaxy properties. Emsellem et al. (2011), van de Sande et al. (2017a), Veale et al. (2017), Brough et al. (2017), Wang et al. (2020) show that the fraction of low $\lambda_{\mathrm{r}}$ galaxies, or slow rotators (SRs), increases with stellar mass, and by $10^{11.3}-10^{11.5} \mathrm{M}_{\odot}$, about half of the galaxies are classified as SR. In addition, Emsellem et al. (2011), Cappellari (2016), Brough et al. (2017) show that most SRs live in high density environments, typical of massive groups or galaxy clusters. However, when galaxies are studied at fixed stellar mass, it is yet unclear whether this environmental trend holds (Brough et al. 2017; Greene et al. 2017; Graham et al. 2019; Wang et al. 2020). Despite this uncertainty, it is well known from optical surveys that visually classified early-type galaxies, red and low star formation rate (SFR) galaxies become more common as we move to high density environments (e.g. Dressler 1980; Peng et al. 2010; Deeley et al. 2017; Davies et al. 2019), even after controlling by stellar mass. Weijmans et al. (2014), Foster et al. (2017), Li et al. (2018a), Krajnović et al. (2018) find that SRs tend to have a higher occurrence of triaxial or prolate intrinsic shapes compared to fast rotators, 
which are mostly oblate, axisymmetric systems (often with bars). The intrinsic stellar populations of SRs indicate flat $\alpha / \mathrm{Fe}$ metallicity radial profiles, uniform old stellar ages, and declining metallicity radial profiles (where the central parts are more metal-rich than the outer parts; Kuntschner et al. 2010; Bernardi et al. 2019; Krajnović et al. 2020).

An outstanding question is what causes morphological or kinematic transformation in galaxies, and whether the same processes are responsible for quenching their star formation. Several simulations have suggested that an effective way of transforming the kinematics of galaxies is via galaxy mergers (e.g. Di Matteo et al. 2009; Jesseit et al. 2009; Bois et al. 2011; Naab et al. 2014; Choi \& Yi 2017; Lagos et al. 2017, 2018a,b; Penoyre et al. 2017; Schulze et al. 2018). Although the exact remnant of a galaxy merger is dependent on many of the merger parameters involved (e.g. mass ratio, gas mass, orbital parameters, etc; e.g. Di Matteo et al. 2009; Naab et al. 2014; Lagos et al. 2018b), some general trends have been reported in the literature. Among the most interesting ones is the fact that gas-poor mergers tend to decrease $\lambda_{\mathrm{r}}$ (Naab et al. 2014; Lagos et al. 2018a), a series of minor mergers or a single major merger can have a similar effect (Naab et al. 2014; Choi \& Yi 2017; Lagos et al. 2018a), and that circular orbits preferentially produce fast rotators (Lagos et al. 2018b; Li et al. 2018b). One common conclusion among simulations is that even if an SR remnant is formed after a merger, continuous accretion and star formation can quickly rebuild the galaxy disc and turn the galaxy into a fast rotator (Naab et al. 2014; Sparre \& Springel 2016; Lagos et al. 2017; Penoyre et al. 2017; Walo-Martín et al. 2020). The latter suggests that quenching either prior or during the kinematic transformation is required to produce an SR. Another possible way of transforming galaxies is via environmental effects, such as interactions between galaxies or with the tidal field of the group or cluster (e.g. Choi \& Yi 2017). With the aim of isolating the effect of environment, Cortese et al. (2019) focused on the relation between the change in SFR and $\lambda_{\mathrm{r}}$ of $z=0$ satellite galaxies in EAGLE since they were accreted, finding no correlation between the two. This suggests that quenching and kinematic transformation are distinct processes (see also Correa, Schaye \& Trayford 2019; Tacchella et al. 2019; Wright et al. 2019 for similar conclusions regarding the connection of quenching with other morphological indicators in simulations).

Many of the conclusions above have been achieved by separating fast and slow rotators using parametric selections in the $\lambda_{\mathrm{r}}-$ $\epsilon$ plane. However, the population of galaxies obtained by these parametric forms is diverse, encompassing galaxies that are likely to have different origins. Those include what would be considered classic ellipticals (round, non-rotating objects), relatively flat SRs (flat, non-rotating objects), prolate galaxies (those that display little rotation and rotate along the minor axis), and $2 \sigma$ galaxies (which have counter-rotating discs that tend to cancel each other's angular momentum yielding a net low rotational velocity) (e.g. Emsellem et al. 2011; Cappellari 2016; van de Sande et al. 2021). In addition, simulations suggest that studying the kinematic properties of galaxies beyond $\lambda_{\mathrm{r}}$ can yield important information regarding the formation histories of galaxies (Bois et al. 2011; Naab et al. 2014; Schulze et al. 2020). van de Sande et al. (2021) analysed $\approx 1800$ SAMI galaxies and compared the visual classification of the kinematic maps of galaxies with how they would be classified if they were to use a parametric selection, finding that no simple parametric cut in the $\lambda_{\mathrm{r}}-\epsilon$ plane can truly provide a high completeness, low contamination sample of galaxies visually classified as non-rotators. The reason why contamination is a lot higher than in the original work of Emsellem et al. (2011) is likely the poorer spatial resolution in SAMI compared to the survey ATLAS ${ }^{3 \mathrm{D}}$ used by Emsellem et al. (2011). Because other large IFS surveys, such as MaNGA, generally have similarly limited spatial resolution, a high contamination in the parametric selection of SRs to SAMI is also expected. This lends significant weight to the process of visual classification if we are to understand the formation mechanisms of truly non- or weakly rotating galaxies and the possible connection between kinematic transformation and quenching.

Very few examples exist of visual kinematic classification of galaxies in simulations. Among these are the work of Li et al. (2018b), who used visual classification of galaxies in the Illustris simulation to find prolate galaxies and study their formation mechanisms. They found that the vast majority of prolate galaxies in their simulation have had galaxy mergers of nearly radial orbits. Ebrová, Łokas \& Eliášek (2021) used visual classification of Illustris galaxies to identify those with kinematically decoupled cores (KDCs) and found that they were long lived, with the vast majority of them forming after major mergers. Schulze et al. (2018) visually classified the kinematic maps of early-type galaxies in the Magneticum simulation, finding a diverse family among SRs, including non-rotators, prolates, and $2 \sigma$ galaxies. Schulze et al. (2018) found that the parametric selection of SRs of Emsellem et al. (2011) led to significant contamination, with many galaxies classed as 'rotators' being misclassified as SR. These works show that visual classification of simulated galaxies can yield new, important information about the formation of galaxies.

In this paper we aim to understand the formation pathways of SRs and possible connection to quenching using the EAGLE simulations. EAGLE is a state-of-the-art cosmological hydrodynamical simulation suite (Crain et al. 2015; Schaye et al. 2015). Its largest cosmological box has a good compromise between volume, $(100 \mathrm{Mpc})^{3}$, and spatial resolution, $700 \mathrm{pc}$, that allows us to have a statistically significant sample of galaxies (several thousands with stellar masses, $M_{\star}>10^{10} \mathrm{M}_{\odot}$ ) and with enough structural detail to be able to study their stellar kinematic properties. EAGLE has been compared to several observations of the structural and kinematic properties of galaxies in observations, finding that the simulation can reproduce reasonably well the size-stellar mass relation of active and passive galaxies across cosmic time (Lange et al. 2016; Furlong et al. 2017; Rosito et al. 2019), the stellar angular momentum-stellar mass relation (Lagos et al. 2017), the fraction of SRs versus stellar mass (Lagos et al. 2018a), and the distribution of stellar rotation-todispersion velocity ratio (van de Sande et al. 2019; Walo-Martín et al. 2020). This makes EAGLE well suited for our experiment. Because we are interested in separating truly SR galaxies from the rest of the galaxies, we go through a similar exercise as van de Sande et al. (2021), and visually inspect galaxies in EAGLE at $z=0$ to (i) select SRs, and (ii) separate different classes of SRs (flat versus round SRs, prolate and $2 \sigma$ galaxies). We then take advantage of the plethora of galaxy properties EAGLE allows us to measure to investigate whether the different merger histories of SRs in EAGLE leave imprints on their intrinsic galaxy properties and kinematic class at $z=0$ that could in principle be used to connect to observed SRs and to understand whether quenching and kinematic transformation happen in tandem or not.

This paper is organized as follows. Section 2 provides a brief summary of the EAGLE simulations, how we compute kinematic properties of galaxies and visually classify them, and build the galaxy merger history of galaxies. We also compare the properties of SRs between the visually selected versus parametric-selected ones in EAGLE. Section 3 analyses the merger history, kinematic transformation and quenching of star formation, and the stellar 
Table 1. Specifications of the EAGLE Ref-L100N1504 simulation used in this paper. The rows list: (1) initial particle masses of gas and (2) dark matter, (3) comoving Plummer-equivalent gravitational softening length, and (4) maximum physical gravitational softening length. Units are indicated in each row. EAGLE adopts (3) as the softening length at $z \geq 2.8$, and (4) at $z$ $<2.8$. This simulation has a side length of $L=100 \mathrm{cMpc}^{3}$. Here, pkpc and ckpc refer to proper and comoving kpc, respectively.

\begin{tabular}{lllc}
\hline & \multicolumn{1}{c}{ Property } & Units & Value \\
\hline$(1)$ & Gas particle mass & $\left(\mathrm{M}_{\odot}\right)$ & $1.81 \times 10^{6}$ \\
$(2)$ & DM particle mass & $\left(\mathrm{M}_{\odot}\right)$ & $9.7 \times 10^{6}$ \\
$(3)$ & Softening length & $(\mathrm{ckpc})$ & 2.66 \\
$(4)$ & max. gravitational softening & $(\mathrm{pkpc})$ & 0.7 \\
\hline
\end{tabular}

populations of the galaxies that are visually classified as SRs in EAGLE. Section 4 analyses the connection between the different kinematic classes of SRs in EAGLE with their merger history, and finally in Section 5 presents a discussion of the main results and our conclusions.

\section{THE EAgLE SIMULATION}

The EAGLE simulation suite (described in detail in Schaye et al. 2015, hereafter S15, and Crain et al. 2015, hereafter C15) consists of a large number of cosmological hydrodynamic simulations with different resolutions, cosmological volumes and subgrid models, adopting a Planck Collaboration XVI (2014) cosmology. S15 introduced a reference model, within which the parameters of the sub-grid models governing energy feedback from stars and accreting black holes (BHs) were calibrated to ensure a good match to the $z=0.1$ galaxy stellar mass function, the sizes of present-day disc galaxies and the $\mathrm{BH}-$-stellar mass relation (see $\mathrm{C} 15$ for details on the tuning of parameters).

Table 1 summarizes the numerical parameters of the simulation used in this work. Throughout the text we use pkpc to denote proper kiloparsecs and cMpc to denote comoving megaparsecs. A key aspect of EAGLE is the use of state-of-the-art subgrid models that capture unresolved physics. The subgrid physics modules adopted by EAGLE include: (i) radiative cooling and photoheating (Wiersma, Schaye \& Smith 2009a), (ii) star formation (Schaye \& Dalla Vecchia 2008), (iii) stellar evolution and chemical enrichment (Wiersma et al. 2009b), (iv) stellar feedback (Dalla Vecchia \& Schaye 2012), and (v) BH growth and active galactic nucleus (AGN) feedback (Rosas-Guevara et al. 2015). In addition, the fraction of atomic and molecular gas in a gas particle is calculated in post-processing following Rahmati et al. (2013) and Lagos et al. (2015). EAGLE employs SUBFIND (Springel et al. 2001; Dolag et al. 2009) to identify self-bound overdensities of particles within haloes (i.e. substructures). These substructures are the galaxies in EAGLE.

Throughout the text we will refer to 'central' and 'satellite' galaxies, where the central corresponds to the galaxy hosted by the main subhalo of a Friends-of-Friends halo, while other subhaloes within the group host satellite galaxies (Qu et al. 2017). Lagos et al. (2018a) computed the stellar spin parameters of galaxies in EAGLE for the simulation of Table 1, using the definition of Emsellem et al. (2007):

$\lambda_{\mathrm{r}}=\frac{\sum_{\mathrm{i}} L_{\mathrm{i}} r_{\mathrm{i}}\left|V_{\mathrm{i}}\right|}{\sum_{\mathrm{i}} L_{\mathrm{i}} r_{\mathrm{i}} \sqrt{V_{\mathrm{i}}^{2}+\sigma_{\mathrm{i}}^{2}}}$,

where $V_{i}$ and $\sigma_{i}$ are the $r$-band luminosity-weighted line-of-sight mean and standard deviation velocities in a pixel $i$ of a cubic grid for each galaxy, and $r_{i}$ is the distance from the centre of the galaxy to the $i$ th pixel (i.e. the circular radius). Each cubic grid is computed using a cell of side $1.5 \mathrm{pkpc}$, which Lagos et al. (2018a) showed produce well-converged results. As in Emsellem et al. (2011), to measure these quantities within $r$, we only include pixels enclosed by the ellipse of major axis $r$, ellipticity $\epsilon(r)$, and position angle $\theta_{\mathrm{PA}}(\mathrm{r}) . \epsilon(r)$ is computed within circular apertures of radii $r$ using the diagonalized inertia tensor of the galaxy's luminosity surface density (see equations 1-3 in Lagos et al. 2018a which follow Cappellari et al. 2007). Here, we adopt $r=r_{50}$, the half-light radius in the $r$ band to make our measurements comparable to observations from local Universe IFU surveys. Note that our method of measuring $\epsilon(r)$ can be biased low compared to what is done in observations, where isophotes are commonly used. More details on how this was computed are presented in section 2.1 of Lagos et al. (2018a). We measure $\lambda_{\mathrm{r}}$ and $\epsilon(r)$ in two orientations: with galaxies viewed through the z-axis of the simulation (considered to be random) and orienting them edge-on (using the stellar specific angular momentum). As we measure both these quantities within $r_{50}$, throughout the text we refer to them as $\lambda_{\mathrm{r}_{50}}$ and $\epsilon_{\mathrm{r}_{50}}$ for random orientations, and $\lambda_{\mathrm{r}_{50} \text {, edge-on }}$ and $\epsilon_{\mathrm{r}_{50}, \text { edge-on }}$ for the edge-on case.

Lagos et al. (2018a) showed that the fraction of SRs (using a variety of definitions based on $\lambda_{\mathrm{r}_{50}}$ and $\epsilon_{\mathrm{r}_{50}}$ ) decreases steeply with decreasing stellar mass, being $\approx 0.1$ at $10^{10} \mathrm{M}_{\odot}$. Considering this and that the quantities above are well converged at stellar masses above $10^{10} \mathrm{M}_{\odot}$ (see appendix A in Lagos et al. 2018a), in this study we focus solely on galaxies above this stellar mass threshold, which results in 3638 galaxies at $z=0$.

\subsection{Galaxy mergers}

We use the merger trees available in the EAGLE data base (McAlpine et al. 2016) to identify galaxy mergers. These merger trees were created using the $D$ - Trees algorithm of Jiang et al. (2014). Qu et al. (2017) described how this algorithm was adapted to work with EAGLE outputs. Galaxies that went through mergers have more than one progenitor, and for our purpose, we track the most massive progenitors of the merged galaxies, and compare the kinematic properties of those with that of the merger remnant. The trees stored in the public data base of EAGLE connect 29 epochs. The time span between snapshots range from $\approx 0.3 \mathrm{Gyr}$ to $\approx 1$ Gyr. Lagos et al. (2017) showed that these time-scales are appropriate to study the effect of galaxy mergers on the specific angular momentum of galaxies, as $\lesssim 1$ Gyr correspond to the merger settling time. Here, we study the merger history from a loockback time of 0 to $10 \mathrm{Gyr}$ of $z=0$ galaxies. We classify galaxy mergers as major mergers when the stellar mass ratio between the secondary and the primary galaxy, $M_{\star, \text { sec }} / M_{\star \text {, prim }}$, is $\geq 0.3$. Minor mergers are those in which this ratio is between 0.1 and 0.3 . We classify mergers with smaller mass ratios as 'very minor mergers'. The distinction between very minor mergers and higher mass ratio mergers is important, as the remnants of the former can have drastically different properties (Karademir et al. 2019). Even with this classification of mergers, $\approx 21$ percent of galaxies with $M_{\star} \geq 10^{10} \mathrm{M}_{\odot}$ do not have mergers identified in the last 10 Gyr. Table 2 summarizes the number of galaxies we find in each of these merger classes.

In addition, we compute the total star-forming gas $\left(M_{\mathrm{SFgas}}\right)$-tostellar mass ratio involved in the galaxy merger: $M_{\mathrm{SFgas}, \text { total }} / M_{\star, \text { total }}=$ $\sum M_{\mathrm{SFgas}}^{i} / \sum M_{\star}^{i}$, where $i=0,1$ (for two galaxies involved in a merger). This fraction provides a measurement of whether a merger is gas-rich or poor, with a threshold at $M_{\mathrm{SFgas}}$, total $/ M_{\star \text {, total }}$ $\approx 0.1$ separating gas-poor and gas-intermediate or rich mergers. This threshold comes from the distribution of $M_{\mathrm{SFgas}}$, total $/ M_{\star \text {, total }}$ in 
Table 2. Number of galaxies in EAGLE at $z=0$ that have $M_{\star} \geq 10^{10} \mathrm{M}_{\odot}$ and that went through $\geq 1$ major mergers in the last $10 \mathrm{Gyr}$; through $\geq 1$ minor mergers and $=0$ major mergers (in the same time period); through $\geq 1$ very minor mergers and $=0$ minor/major mergers (in the same time period); have not had any mergers in the last $10 \mathrm{Gyr}$; have had $\geq 1$ dry mergers; have had no dry mergers, but $\geq 1$ wet mergers.

\begin{tabular}{lc}
\hline Sample & Number \\
\hline All $M_{\star} \geq 10^{10} \mathrm{M}_{\odot}$ & 3638 \\
Major mergers & 1113 \\
Minor mergers (and no major mergers) & 1042 \\
Very minor mergers (and no minor/major mergers) & 708 \\
w/o mergers & 775 \\
Dry mergers $\left(M_{\star, \text { sec }} / M_{\star, \text { prim }}>0\right.$ ) & 650 \\
Wet mergers (no dry mergers; $\left.M_{\star, \text { sec }} / M_{\star, \text { prim }}>0\right)$ & 2213 \\
\hline
\end{tabular}

galaxy mergers in EAGLE presented by Lagos et al. (2018b). We split galaxies between those that went through dry and wet mergers, by selecting those that had $\geq 1$ dry mergers over the last $10 \mathrm{Gyr}$, and those that did not but had $\geq 1$ wet mergers over the same period (statistics of those are presented in Table 2). The logic of this split is that dry mergers on average happen later compared to wet mergers, and hence in the presence of dry mergers, the past history of wet mergers is less relevant.

\subsection{Building mock kinematic maps of EAGLE galaxies}

An important aspect of this paper is the visual classification of EAGLE galaxies in a way that resembles the SAMI survey classification of van de Sande et al. (2021). Hence, we aim to build stellar kinematic maps that mimic SAMI in terms of spatial and velocity sampling, as well as seeing. For this purpose we generate mock kinematic cubes for each EAGLE galaxy with $M_{\star} \geq 10^{10} \mathrm{M}_{\odot}$ using the R-package SIMSPIN (Harborne, Power \& Robotham 2020a).

SIMSPIN takes an N-body or hydrodynamical SPH simulation and produces a kinematic data cube in the style of an IFS observation. We have designed these mock observations to reflect the observational parameters of the SAMI survey (Scott et al. 2018): kinematic cubes have a spatial pixel size of 0.5 arcsec and a velocity pixel size of $65 \mathrm{~km} \mathrm{~s}^{-1}$ (Green et al. 2018).

In each case, the stellar particle properties (initial mass, age, and metallicity) are used to assign a flux to each particle. We logarithmically interpolate the GALEXEV synthesis models (Bruzual \& Charlot 2003, hereafter BC03) for simple stellar populations to generate a spectral energy distribution (SED) for each stellar particle using PROSPECT (Robotham et al. 2020). In cases in which the metallicities lie outside the boundaries of the $\mathrm{BC} 03$ range, we extrapolate to find a solution as in Trayford et al. (2015).

Each galaxy has been projected to a distance such that the projected half-stellar mass radius is equivalent to a consistent number of pixels within the aperture to reduce the effects of spatial sampling. The velocities of each particle have been convolved with a Gaussian function to mimic the instrumentation effects, using a kernel of $2.65 \AA$ to match the line-spread function of the blue observing arm of the SAMI spectrograph (van de Sande et al. 2017b). We have further included a realistic level of seeing in these mock observations by convolving each spatial plane in the data cube with a Gaussian point-spread function with full width at half-maximum (FWHM) of 1 arcsec. These images are produced at several inclinations, oriented using the inertia tensor. Unless otherwise specified, we use the images produced at an inclination of 60 degrees. This inclination is chosen as we are trying to balance two requirements: (i) to avoid edge-on inclinations as those hamper the visual classification of, specially, velocity dispersion maps when searching for decoupled cores, local peaks of $\sigma$, etc.; (ii) to avoid orientations too close to face-on as those would make all galaxies appear round. The chosen 60 degrees is a good compromise, corresponds to the average inclination of galaxies in the Universe, and is one in which intrinsically flat galaxies are still easy to identify as such.

Flux, line-of-sight (LOS) velocity, and velocity dispersion maps are constructed from these mock data cubes and visualized using PYNMAP. ${ }^{1}$ Flux maps are simply the sum of the flux in each pixel throughout the cube; LOS velocity maps are the flux-weighted mean of the velocities at each pixel; and LOS velocity dispersion maps are the flux-weighted standard deviation of the velocities in each pixel. For more information about the construction of these data products, we direct the reader to Harborne et al. (2020a). Fig. 1 shows examples of the maps generated with SIMSPIN and visualized using PYNMAP. In some cases the central stellar velocity dispersion is lower than in the outskirts (see third and bottom right-hand panels of Fig. 1). We find this to be a frequent feature in massive galaxies in EAGLE. In fact, $\approx 55$ per cent of galaxies with $M_{\star}>10^{10} \mathrm{M}_{\odot}$ have $\sigma_{\star}\left(0.5 \mathrm{r}_{50}\right)<\sigma_{\star}\left(\mathrm{r}_{50}\right)$, where $\sigma_{\star}\left(0.5 \mathrm{r}_{50}\right)$ and $\sigma_{\star}\left(r_{50}\right)$ are the stellar velocity dispersions measured using particles within $0.5 \mathrm{r}_{50}$ and $\mathrm{r}_{50}$, respectively. This is further discussed in Section 3.3.

\subsection{Visual classification of simulated kinematic maps}

In previous papers we have classified galaxies as slow and fast rotators using parametric criteria based on the distribution of galaxies in the $\lambda_{\mathrm{r}_{50}}-\epsilon_{\mathrm{r}_{50}}$ plane. Recently, van de Sande et al. (2021) have questioned the applicability of these criteria which, for the most part, have been built with higher resolution data, highlighting that a visual classification of kinematic maps yields different classifications to those obtained by parametric criteria. Harborne et al. (2020b), using numerical simulations of galaxies of different disc/bulge ratios, quantified how resolution affects the derived $\lambda_{R}$. They found that lower resolution leads to artificially low $\lambda_{R}$, which can lead to galaxies being misclassified as being below the line of slow rotators in the $\lambda_{\mathrm{R}}-\epsilon$ plane. A similar result was presented in Graham et al. (2018). In addition, Naab et al. (2014) showed that the details of the kinematic maps of galaxies can yield important information about the formation history of SRs, making visual classification desirable to advance our understanding of galaxy evolution.

Here, we take advantage of the maps generated in Section 2.2 to go through a similar classification campaign as presented in van de Sande et al. (2021) for SAMI. The aim is to isolate 'unambiguous' SRs in EAGLE and understand their relation to assembly history as well as environment. Here, unambiguous refers to galaxies that visually look like SRs. We first select all galaxies with $M_{\star} \geq$ $10^{10} \mathrm{M}_{\odot}$, which are expected to have well-converged internal stellar kinematics. From this sample, we take a very conservative selection in $\lambda_{\mathrm{r}_{50}, \text { edge-on }} \leq 0.2$. van de Sande et al. (2021) decomposed the galaxy population in bins of stellar mass and used mixture models to determine the existence of a distinct population of low $\lambda_{\mathrm{r}_{50}}$ in SAMI and several simulations, including EAGLE. A cut at $\lambda_{\mathrm{r}_{50} \text {,edge-on }} \leq 0.2$ comfortably includes all galaxies that belong to the population of low $\lambda_{\mathrm{r}_{50}}$ in EAGLE. This selection in stellar mass and $\lambda_{\mathrm{r}_{50} \text {,edge-on }}$ yields 559 galaxies at $z=0$.

We ask five members of our team to independently classify those maps into six different kinematic classes: flat SRs (FSR), round SRs

\footnotetext{
${ }^{1}$ https://github.com/emsellem/pynmap
} 

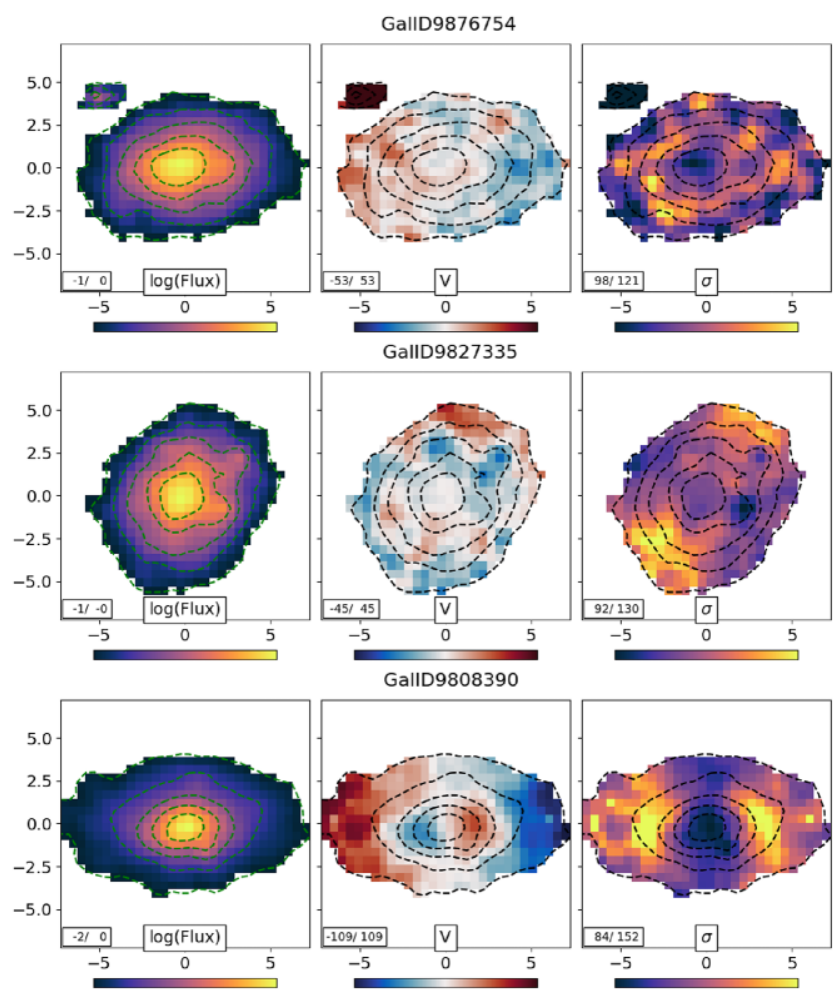

GallD8295057
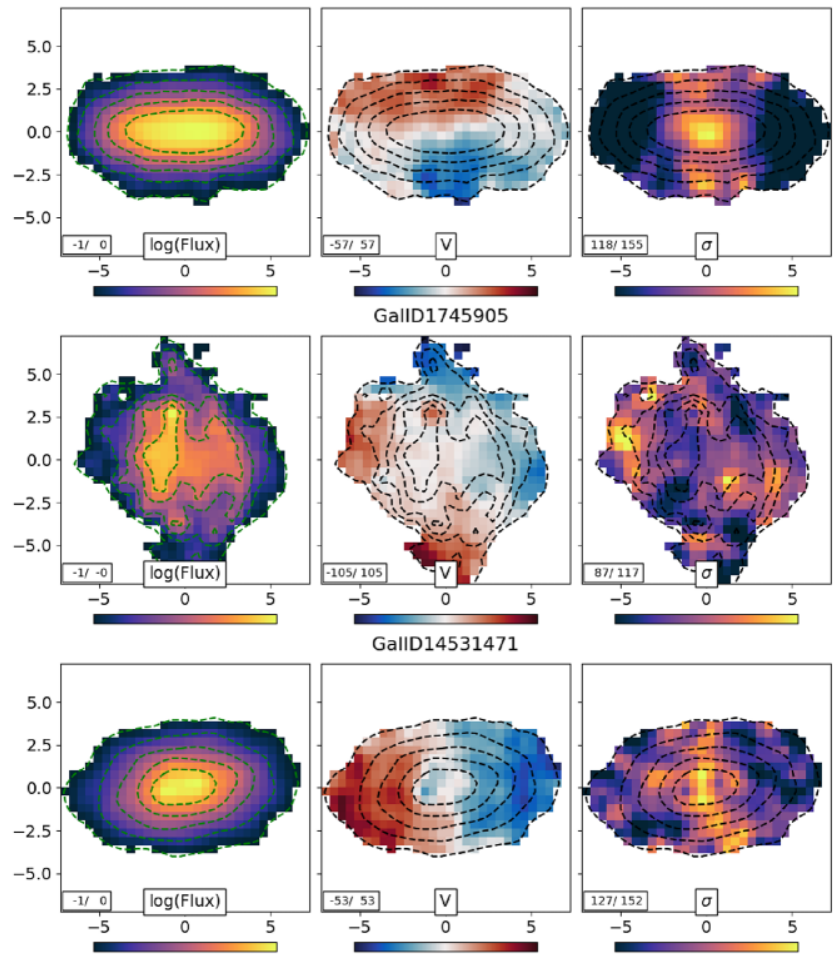

Figure 1. Examples of flux (left-hand panel), LOS stellar velocity (middle) and velocity dispersion (right-hand panel) maps for $z=0$ galaxies in EAGLE. Units in the $\mathrm{x}$ - and $\mathrm{y}$-axes are pkpc. Colour bar's minima and maxima are shown at the bottom left of each panel (with velocities in $\mathrm{km} \mathrm{s}^{-1}$ ). From top to bottom, we show examples of galaxies with 100 per cent agreement among classifiers that belong to the flat SR, round SR, $2 \sigma$, prolate, unclear and rotator kinematic classes, respectively (see Section 2.3 for details). The Galaxy ID is shown at the top of each row, and can be matched to the IDs in the EAGLE data base (McAlpine et al. 2016).
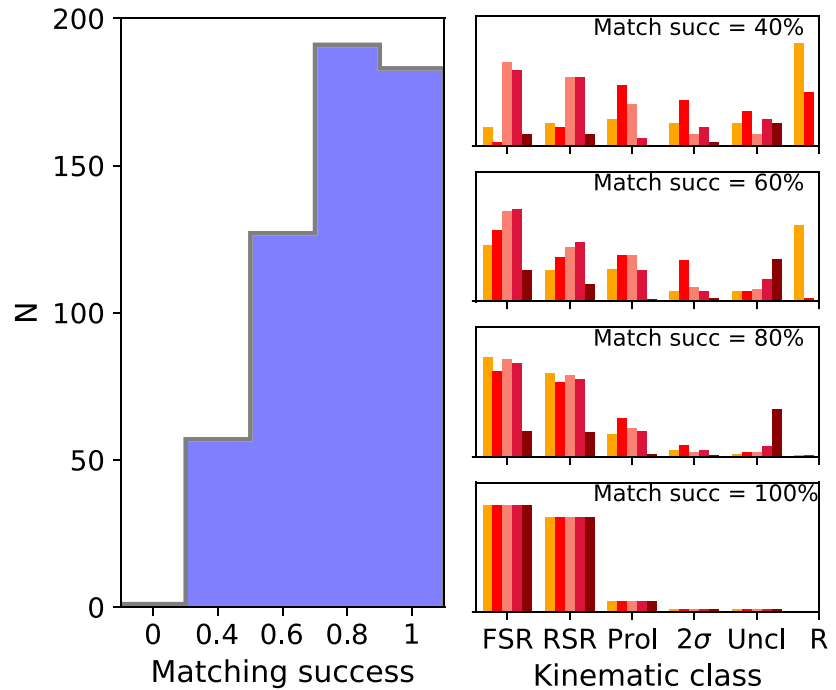

Figure 2. Left-hand panel: Distribution of the matching success, with 0 per cent indicating no agreement between the kinematic classes of classifiers. The majority of galaxies have an agreement of $\geq 60$ percent among classifiers. Right-hand panels: Probability density function of kinematic classes in four bands of matching success, as labelled in each panel, with each colour showing a different classifier.

(RSR), $2 \sigma$ galaxies (that display two clear peaks in the stellar velocity dispersion map), prolate galaxies (Prol; those displaying rotation along the minor axis), unclear (Uncl) and rotators. We purposely avoid giving any instructions to the classifiers and simply let them assess what they expect for these different classes. We believe this provides a truly independent classification and avoid confirmation bias. We then compiled these classifications and analyse the level of agreement. Fig. 1 shows six examples of the kinematic classes above, for which all classifiers agreed. For the Uncl cases, we find that those generally are similar to the example shown in Fig. 1, in which there is a lot of substructure that is assigned to the same subhalo. This is a well-known shortcoming of 3D subhalo finders (Cañas et al. 2019), which tends to get worse in high density environments.

The left-hand panel of Fig. 2 shows the distribution of matching success among classifiers. Most galaxies can be kinematically classified with an agreement $\geq 60$ per cent ( 3 out of 5 classifiers agree on the class). By adopting this threshold, we are left with 501 of the initially 559 classified galaxies (i.e. $\approx 90$ per cent of the sample). The right-hand panel of Fig. 2 shows the distribution of kinematic classes in the different levels of agreement of each independent classifier. For the cases in which $2 / 5$ agree, we find that the conflict arises in whether galaxies are rotators/unclear or not. $2 \sigma$ galaxies are also hard to classify, with most of them being in the matching success panels of 40 per cent and 60 per cent. We note that EAGLE produces galaxies of diverse kinematic classes, which are also seen in observations (Emsellem et al. 2011). Schulze et al. (2018) via visual classification of the kinematic maps of early-type galaxies in the Magneticum simulations also found similarly diverse kinematic classes. Table 3 presents the number of galaxies classified in each kinematic class with a confidence level $\geq 60$ per cent.

The classification between FSR and RSR so far adopted can be subjective. In order to determine whether there is an obvious ellipticity threshold distinguishing between the two subclasses, we turn to the ellipticity distribution of the visual classes, FSRs and RSRs. This is shown in Fig. 3 for each classifier. Overall, a threshold 
Table 3. Number of galaxies at $z=0$ visually classified with a confidence $\geq 60$ per cent in each kinematic class.

\begin{tabular}{lc}
\hline Sample & $N$ \\
\hline All (confidence $\geq 60$ per cent) & 501 \\
Flat SR & 238 \\
Round SR & 192 \\
Prolate & 49 \\
$2 \sigma$ & 12 \\
Unclear & 9 \\
Rotator & 1 \\
\hline
\end{tabular}

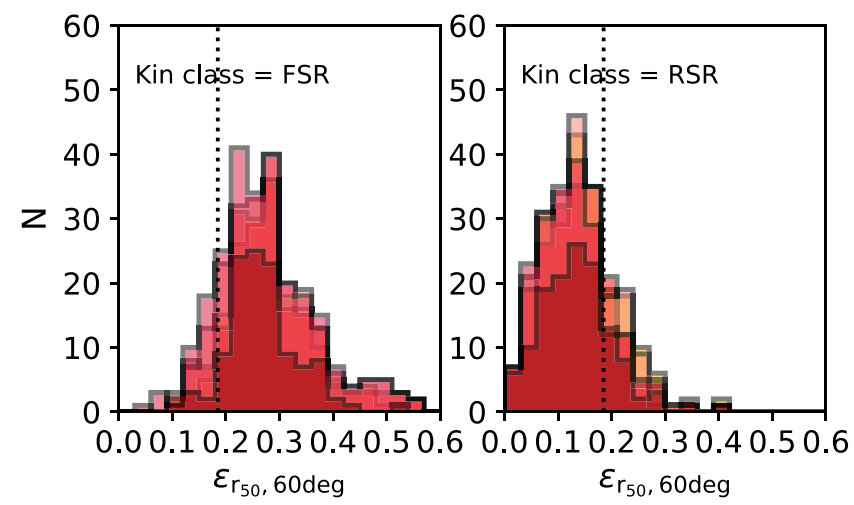

Figure 3. Distribution of ellipticities for flat (left-hand panel) and round (right-hand panel) SRs. Each coloured histogram shows a different classifier. Ellipticities here are measured directly from the SIMSPIN maps, which adopted an inclination of 60 degrees. The vertical line shows $\epsilon_{\mathrm{r}_{50}, 60 \mathrm{deg}}=0.2$, which we consider a reasonable threshold to separate flat and round SRs.

Table 4. Number of galaxies in our unambiguous SR sample at $z=0$ that went through $\geq 1$ major mergers in the last $10 \mathrm{Gyr}$; through $\geq 1$ minor mergers and $=0$ major mergers (in the same time period); through $\geq 1$ very minor mergers and $=0$ minor/major mergers (in the same time period); have not had any mergers in the last $10 \mathrm{Gyr}$; have had $\geq 1$ dry mergers; have had no dry mergers, but $\geq 1$ wet mergers. We also show the breakdown between centrals and satellites in each group.

\begin{tabular}{lccc}
\hline Sample & All & Cens & Sats \\
\hline Visual SRs (confidence $\geq 60$ per cent) & 479 & 293 & 186 \\
Major mergers & 225 & 149 & 76 \\
Minor mergers (and no major mergers) & 145 & 87 & 58 \\
Very minor mergers (and no minor/major mergers) & 72 & 41 & 31 \\
w/o mergers & 37 & 16 & 21 \\
dry mergers & 178 & 113 & 65 \\
Wet mergers (no dry mergers) & 264 & 164 & 100 \\
\hline
\end{tabular}

of $\epsilon_{\mathrm{r}_{50}, 60 \mathrm{deg}}=0.2$ appears appropriate for all classifiers. From hereon, we use this threshold to classify galaxies between FSR and RSR.

From these findings, we will consider as SRs in EAGLE all galaxies visually classified as FSR, RSR, and prolate. Unless otherwise specified, we only consider SRs in which there is $\geq 60$ percent agreement among classifiers and refer to this sample as unambiguous SRs. This threshold was chosen to be similar to that adopted in van de Sande et al. (2021). Table 4 presents the breakdown in the incidence of different types of mergers in the unambiguous SRs and the breakdown between centrals and satellites. Because some of the subsamples are rather small, we tend to subdivide them in ways that we always have $\geq 10$ galaxies to measure medians from.

\subsection{Parametric versus visually classified slow rotators in EAGLE}

As discussed in the introduction, most simulation-based papers have adopted a parametric selection of SRs to analyse their formation history. Thus, it is important to understand how different our visual classification of SRs is from parametric selections.

Fig. 4 shows the distribution of $\lambda_{\mathrm{r}_{50}}, \epsilon_{\mathrm{r}_{50}}, \sigma_{\mathrm{r}_{50}}$, and stellar mass of all galaxies in EAGLE at $z=0$ with $M_{\star} \geq 10^{10} \mathrm{M}_{\odot}$, and the subsamples of galaxies selected as SRs based on the parametric classification of van de Sande et al. (2021) and the visually identified SRs. The parametric classification of van de Sande et al. (2021) is as follows:

$\lambda_{\mathrm{r}_{50}}<0.12+0.25 \epsilon_{\mathrm{r}_{50}}$, for $\epsilon_{\mathrm{r}_{50}} \leq 0.5$.

Most visually classified SRs fall within the classification of van de Sande et al. (2021) with a small fraction $(\approx 12$ per cent) falling in the region of low $\epsilon_{\mathrm{r}_{50}}$ and elevated $\lambda_{\mathrm{r}_{50}}$. We visually inspect the galaxies that are in the unambiguous SR sample and have $\lambda_{\mathrm{r}_{50}}>0.2$ ( $\approx 6$ percent of the sample). We find these are a mix bag of $2 \sigma$ galaxies, galaxies that have some rotation in the outskirts but none in the central parts, and galaxies that have contamination from substructure but not enough as to fall in the 'unclear' category, so they can still be easily identified as SR. Something in common among these galaxies is that they have $0.1<\lambda_{\mathrm{r}_{0} \text {, edge-on }}<0.2$, so by the original criterion of Emsellem et al. (2007) they would not be considered SRs. There is another, even smaller fraction $(\approx 2.7$ per cent $)$ of SRs in the unambiguous SR sample with $\epsilon_{\mathrm{r}_{50}}>0.5$. The success rate of the van de Sande et al. (2021) classification in EAGLE is $\approx 85$ per cent, which is similar to the success rate obtained by the authors using a visually classified sample of SAMI galaxies $(\approx 90$ per cent $)$. The downside is that this parametric selection has a high contamination rate, selecting 295 galaxies that are not SRs (40 of those have a lower visual classification confidence, $<60$ per cent, and 205 have $\lambda_{\mathrm{r}_{50} \text {, edge-on }}>0.2$ ). Even in the best case scenario (in which we drop our confidence threshold down to 40 percent), the purity of the selection (fraction of unambiguous SRs) would be 65 percent in EAGLE.

In general, we find that the visually classified SRs prefer $\epsilon_{\mathrm{r}_{50}} \lesssim 0.5$, with most of them having $0.1 \lesssim \epsilon_{\mathrm{r}_{50}} \lesssim 0.5$. Note that these values of $\epsilon_{\mathrm{r}_{50}}$ cover a wider range than the SR selection criterion of Cappellari (2016), who imposes a threshold $\epsilon_{\mathrm{r}_{50}} \leq 0.4$ for a galaxy to be considered an SR. Thus, the criterion of van de Sande et al. (2021) works better in EAGLE, albeit with a high contamination. From the first and third panels of Fig. 4, it is clear that visually classified SRs tend to populate the lower $\lambda_{\mathrm{r}_{50}}$ and higher $\sigma_{\mathrm{r}_{50}}$ regions of the parametric SRs distributions. There is also a small tendency of the visual SRs to have lower $\epsilon_{\mathrm{r}_{50}}$ and higher stellar masses than the parametric SRs. In addition to the properties in Fig. 4, we investigated several other galaxy properties and found that the specific SFR (sSFR) and $r_{50}$ were on average 23 per cent lower and 10 percent larger, respectively, in the visual SRs compared to the parametric ones. Visual SRs also have a higher incidence of galaxy mergers, with the mean number of mergers in this sample being $\approx 4.3$ compared to 3.7 in the parametric SRs. All the evidence above shows the importance of the visual classification of the kinematic maps we present in this paper required to isolate a sample of unambiguous SRs in the simulation, from which we can study their kinematic transformation.

An interesting result in Fig. 4 regarding the entire galaxy population in EAGLE, is that the $\lambda_{\mathrm{r}_{0}}$ distribution shows signs of a bimodality, with peaks at $\approx 0.2$ and $\approx 0.6$. van de Sande et al. (2021) present a detailed quantification of the existence of a bimodality in $\lambda_{r_{50}}$ at fixed stellar mass, and conclude that even though this bimodality is 

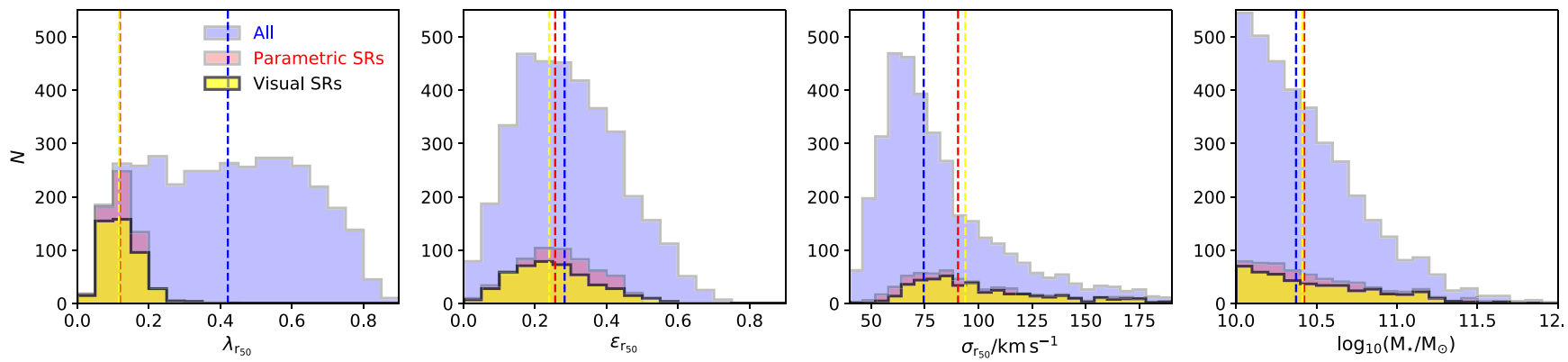

Figure 4. Distribution of $\lambda_{\mathrm{r}_{0}}, \epsilon_{\mathrm{r}_{0}}$, stellar velocity dispersion, and stellar mass of $z=0$ galaxies in EAGLE with $M_{\star} \geq 10^{10} \mathrm{M}_{\odot}$. The distributions are shown for all galaxies, SRs classified following the parametric selection of van de Sande et al. (2021), and the visually classified SRs (with a confidence $\geq 60$ per cent), as labelled. The vertical lines show the medians of each distribution.

clear in SAMI (see also Graham et al. 2018 for a similar analysis in MaNGA), it appears less clear in EAGLE. For massive galaxies, van de Sande et al. (2021) showed that, although two beta functions were required for a good fit, the one peaking at low $\lambda_{\mathrm{r}_{50}}$ in EAGLE had a prominent tail towards high values of $\lambda_{\mathrm{r}_{50}}$. One important difference with the analysis of van de Sande et al. (2021) is that here we include all EAGLE galaxies with $M_{\star} \geq 10^{10} \mathrm{M}_{\odot}$, while van de Sande et al. (2021) analysed a subsample of the simulation selected to have the same stellar mass distribution as the SAMI survey, which ends up biased towards high masses (with a peak at $10^{10.3} \mathrm{M}_{\odot}$ ). This possibly means that the bimodality in observations may be stronger than reported, in which case a volume complete sample would be needed to confirm that. Another important result from Fig. 4 is that the sample of visually classified SRs in EAGLE is only a fraction of the galaxies that would be associated with the low $\lambda_{\mathrm{r}_{50}}$ population. This population of SRs is not distinct enough to be cleanly separated by statistical means, lending support to our approach of visually classifying galaxies to study the formation mechanisms of SRs in EAGLE.

\section{THE PROPERTIES OF SLOW ROTATORS IN EAGLE}

In this section we analyse various properties of EAGLE galaxies selected as SRs based on the visual classification presented in Section 2.3 and that have a classification confidence $\geq 60$ per cent.

We study the distribution of SRs in the $\lambda_{r_{50}}-\epsilon_{\mathrm{r}_{50}}$ in Fig. 5. We separate SRs that had $\geq 1$ minor or major mergers in the last $10 \mathrm{Gyr}$, from those that did not. We show for reference the parametric SR classifications of Cappellari (2016) and van de Sande et al. (2021). Fig. 5 shows that the most massive SRs have had $\geq 1$ minor or major mergers in the last $10 \mathrm{Gyr}$, while in the subset of SRs without mergers or exclusively very minor mergers, we preferentially find lower mass galaxies. This is quantified in the top panel of Fig. 6, where we show the contribution of the four subsets of SRs selected based on their merger history as a function of stellar mass. The subset of 'no mergers' is only present at $10^{10} \mathrm{M}_{\odot}<\mathrm{M}_{\star}<10^{10.5} \mathrm{M}_{\odot}$, while most galaxies in the 'very minor mergers' subset are preferentially in the $10^{10} \mathrm{M}_{\odot}<\mathrm{M}_{\star}<10^{11} \mathrm{M}_{\odot}$ range. The fraction of SRs that have not experienced mergers is much smaller than the 'no mergers' fraction of the entire galaxy population at fixed stellar mass (see bottom panel of Fig. 6). On the other hand, about 40-50 per cent of SRs had $\geq 1$ major mergers in the last $10 \mathrm{Gyr}$, even at relative low stellar masses $\left(10^{10} \mathrm{M}_{\odot}<\mathrm{M}_{\star}<10^{10.5} \mathrm{M}_{\odot}\right)$, which is twice the incidence of major mergers seen in the overall galaxy population at the same stellar mass.

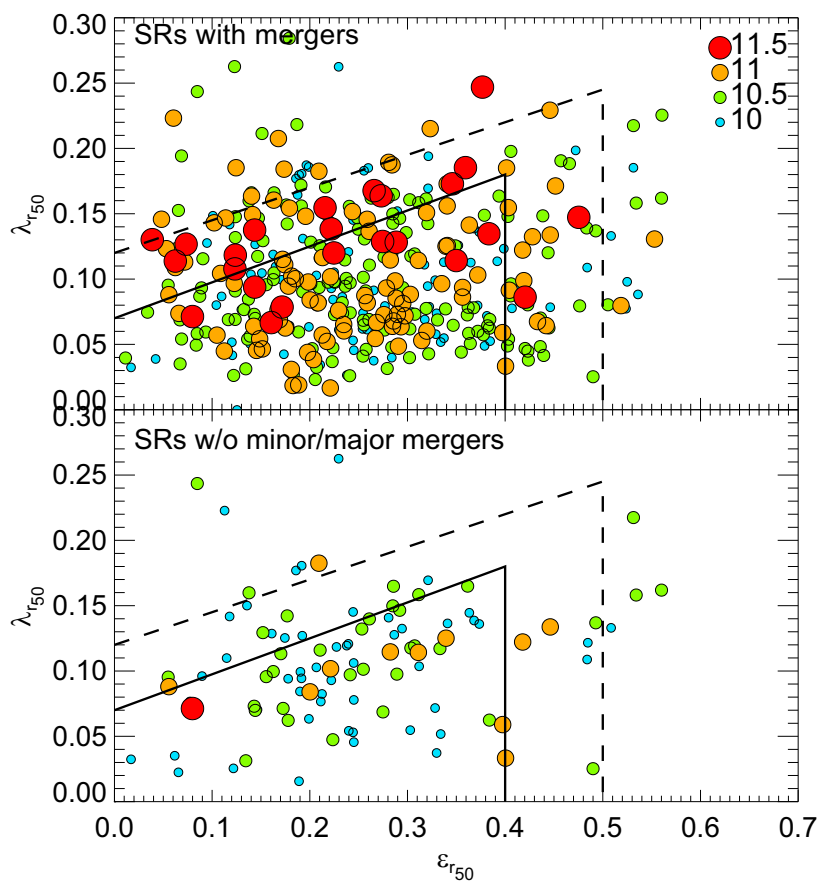

Figure 5. $\lambda_{\mathrm{r}_{50}}$ as a function of $\epsilon_{\mathrm{r}_{50}}$ at $z=0$ for SRs in EAGLE. The top panel shows SRs that have had mergers with mass ratios $\geq 0.1$ in the last $10 \mathrm{Gyr}$, while the bottom panel shows the complement SRs. Sizes and colours of the symbols correspond to different stellar masses, as labelled in the top panel. For reference we show as solid and dashed lines the parametric classifications of Cappellari (2016) and van de Sande et al. (2021), respectively.

Minor and very minor mergers are represented in similar fractions in the SRs and all galaxies samples at fixed stellar mass.

The significantly lower fraction of 'no mergers' and higher fraction of major mergers among SRs shows the importance of the latter in producing SRs in EAGLE. In the coming sections, we analyse intrinsic properties of SRs selected by their merger history to understand whether there are observable properties that are expected to be systematically different among these SRs.

\subsection{Intrinsic properties of slow rotators}

We focus on intrinsic properties of SRs that have attracted interest in the literature, including: intrinsic shape, velocity anisotropy, and sizes. In addition, as we are interested in quenching and galaxy 


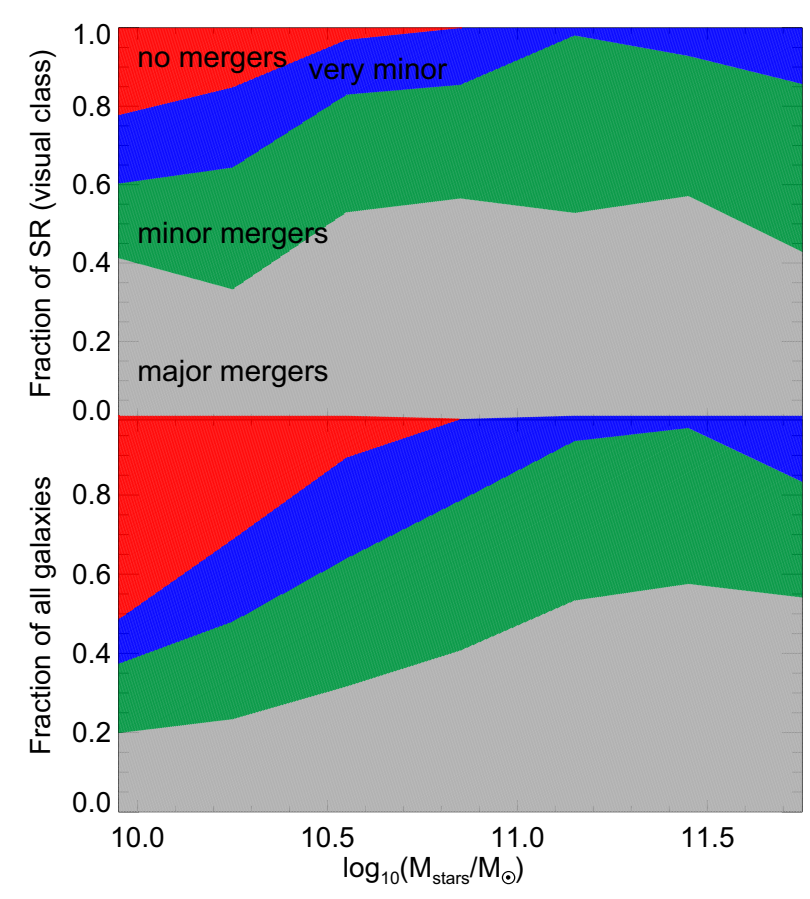

Figure 6. The top panel shows the fraction of $\mathrm{SRs}$ that had $\geq 1$ major mergers (grey shaded region), no major mergers but $\geq 1$ minor mergers (green), no minor or major mergers, but $\geq 1$ very minor mergers (blue) and no mergers (red) over the last $10 \mathrm{Gyr}$, as a function of stellar mass. The bottom panel shows the same but for all galaxies regardless of their kinematic class.

mergers in SRs, we also explore their BH masses and stellar ex-situ fraction, $f_{\text {ex-situ }}$.

The left-hand panels of Fig. 7 show the triaxiality, stellar velocity anisotropy, $f_{\text {ex-situ }}, r$-band half-light radius and $\mathrm{BH}$-to-stellar mass ratio, as a function of stellar mass of SRs at $z=0$ in EAGLE classified based on their merger history. The first two quantities above come from the EAGLE analysis of Thob et al. (2019), which we briefly describe here. For each galaxy, all stellar particles within a spherical aperture of radius $30 \mathrm{pkpc}$ are used to measure the tensor of the quadrupole moments of the mass distribution (which share eigenvectors with the inertia tensor). The axes lengths $a$ (major axis), $b$ (intermediate axis), and $c$ (minor axis) are defined by the square root of the eigenvalues of the mass distribution tensor, $\lambda_{\mathrm{i}}$ (for $i=0,1,2)$. These axes are then used to measure a first pass for the ellipticity $(\epsilon=1-c / a)$ and triaxiality $\left(T=\left(a^{2}-b^{2}\right) /\left(a^{2}-\right.\right.$ $\left.c^{2}\right)$ ). These values are then used to select stellar particles that are enclosed in the ellipsoid of axes ratios $a / b, a / c$ of equal volume as the sphere of $r=30 \mathrm{pkpc}$. These particles are used to remeasure the ellipsoid axes. This iterative process continues until changes in $a$, $b, c$ are $<1$ per cent. A perfect spherical galaxy has $\epsilon=0$ and $T$ is undefined. Low and high values of $T$ correspond to oblate and prolate ellipsoids, respectively. The stellar velocity anisotropy, $\delta_{\text {stars }}$, depends on the velocity dispersion parallel, $\sigma_{\|}$, and perpendicular, $\sigma_{\perp}$, to the stellar angular momentum vector of the galaxy (all measured with stellar particles at $r<30 \mathrm{pkpc}$ from the centre of potential), $\delta_{\text {stars }}=$ $1-\left(\sigma_{\perp} / \sigma_{\|}\right)^{2}$. If $\delta_{\text {stars }}>0$, then the stellar velocity dispersion is dominated by disordered motions in the disc plane.

We also make use of the stellar ex-situ fractions, $f_{\text {ex-situ }}$ computed by Davison et al. (2020) for EAGLE galaxies at $z=0$. Here, $f_{\text {ex-situ }}$ refers to the fraction of stars that did not form in the main progenitor branch of the $z=0$ galaxy, and hence was acquired from galaxies that
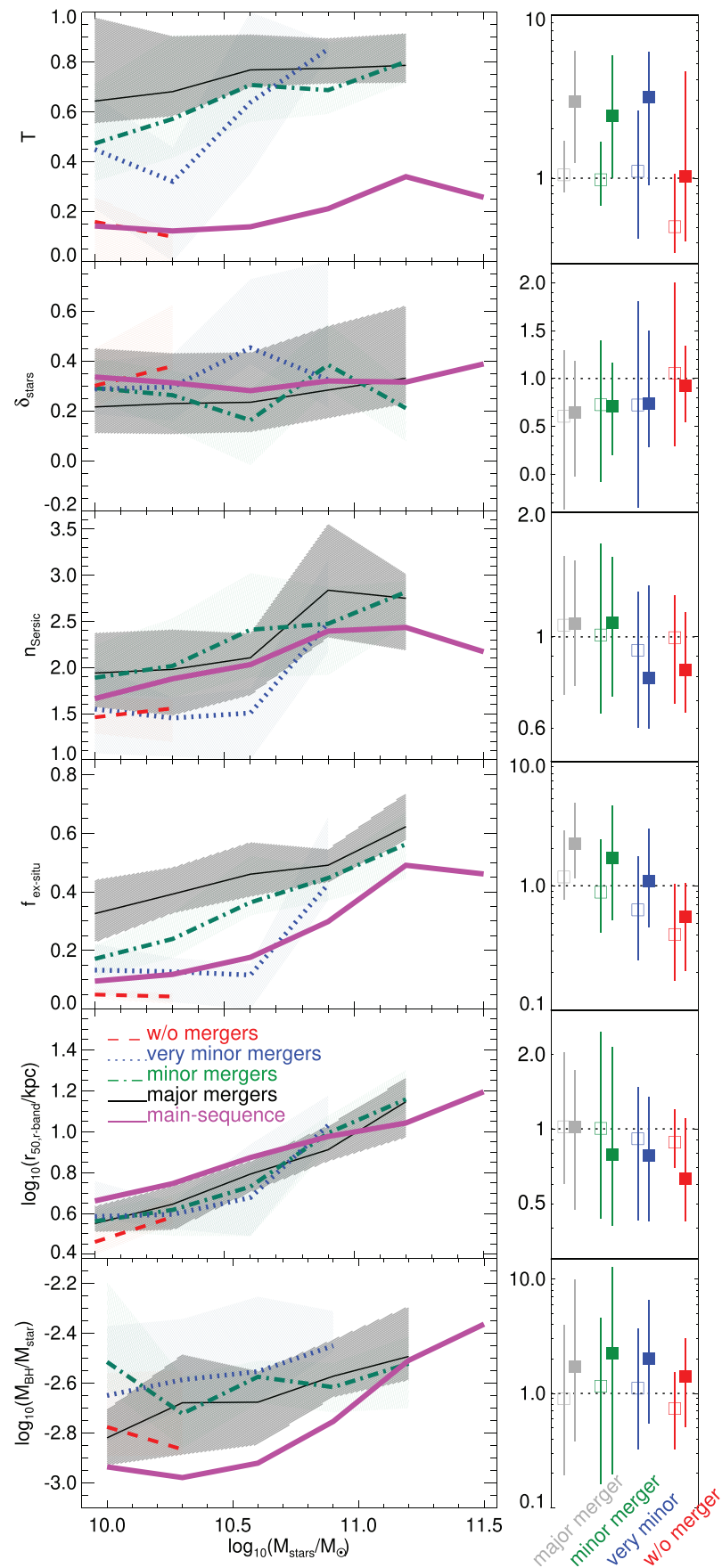

Figure 7. Left-hand panels: Triaxiality (top), anisotropy stellar velocity dispersion (second), 3D Sérsic index (third), ex-situ stellar fraction (fourth), half-light radius (fifth), and BH-to-stellar mass ratio (bottom) as a function of stellar mass for SRs at $z=0$ in EAGLE. We show separately SRs that have had $\geq 1$ major mergers (solid lines), $=0$ major but $\geq 1$ minor mergers (dotdashed lines), $=0$ major/minor mergers but $\geq 1$ very minor mergers (dotted lines), and $=0$ mergers (dashed lines) in the last $10 \mathrm{Gyr}$. Lines with shaded regions show the median and 25th-75th percentile range, respectively, and we only show bins with $\geq 10$ galaxies. For reference, the thick, magenta line shows the median relation for main sequence galaxies in EAGLE (those with an sSFR $\left.>0.01 \mathrm{Gyr}^{-1}\right)$. Parameters are calculated considering all their stellar particles within the inner $30 \mathrm{pkpc}$. Right-hand panels: median and $25 \mathrm{th}-75 \mathrm{th}$ percentile range of the ratio between the properties in the left-hand panel for the four different SRs subsamples of the left-hand panels selected based on their merger history, and two stellar-mass matched samples of fast rotators (filled squares) and SRs (empty squares). The horizontal dotted line marks equality. 
merged on to the main progenitor in the past (or were acquired after close interactions). This is computed considering all stellar particles within $30 \mathrm{kpc}$ from the galaxy's centre. For reference, the left-hand panels of Fig. 7 also show the median of these quantities as a function of stellar mass for galaxies that are considered to be main sequence (those with a sSFR $>0.01 \mathrm{Gyr}^{-1}$; Furlong et al. 2015).

The samples of SRs split by their merger history can quickly become very small and hence the correlation with stellar mass can be noisy. To try to identify main trends, we also show in the right-hand panels of Fig. 7 the median ratio between the quantity in the lefthand panel for the subsample of SRs and for a control sample of fast rotators and SRs matched to have the same stellar mass distribution. ${ }^{2}$

We find that SRs without mergers are very oblate $(T \lesssim 0.2)$ compared to other SRs, and in fact similar to what we expect for main sequence galaxies and fast rotators of the same stellar mass. Even though these SRs are very compact, $r_{50} \approx 2-3 \mathrm{pkpc}$, they are still above the resolution limit by a factor of $\approx 3-4$, and given their mass, we expect them to be resolved with $\gtrsim 3000$ particles, so we consider these measurements reliable.

There is a tendency for $T$ to increase going from SRs that went exclusively through very minor mergers to those that went through major mergers at fixed stellar mass. Most of the prolate SRs $(T \gtrsim$ $0.7)$ correspond to galaxies that went through major mergers, while very minor and minor mergers are preferentially associated with triaxial systems $(0.3 \lesssim T \lesssim 0.7)$, particularly at $10^{10} \mathrm{M}_{\odot} \lesssim \mathrm{M}_{\star}<$ $10^{10.7} \mathrm{M}_{\odot}$. Compared to other simulations we find some interesting differences. Pulsoni et al. (2020) found that in the Illustris-TNG100 simulation there is a large fraction $(\approx 83$ percent $)$ of SRs that are triaxial $(0.3 \lesssim T \lesssim 0.7)$ at $r<1-2 \mathrm{r}_{50}$, which we do not see in EAGLE $(\approx 34$ per cent are triaxial at small radii). Most of the triaxial SRs in Illustris-TNG are in the stellar mass range $10^{10.5}-10^{11.5} \mathrm{M}_{\odot}$, while in EAGLE, 50 per cent ( 80 per cent) are $<10^{10.5} \mathrm{M}_{\odot}\left(10^{10.9} \mathrm{M}_{\odot}\right)$. The reasons for these differences are not easy to pinpoint but it is worth highlighting them for future research.

Most SRs have $0.2 \lesssim \delta_{\text {stars }} \lesssim 0.6$, which is similar to the values reported for SRs in Schulze et al. (2018) for the Magneticum simulations. Most of the galaxies with $\delta_{\text {stars }}<0.2$ are main sequence galaxies (with sSFR $\gtrsim 0.025 \mathrm{Gyr}^{-1}$ ) and fast rotators $\left(0.2 \lesssim \lambda_{\mathrm{r}_{50}} \lesssim\right.$ 0.7 , where the limits correspond to the 25 th -75 th percentile range) also in agreement with the findings in Schulze et al. (2018). We identify a weak trend of $\delta_{\text {stars }}$ increasing when going from SRs that went through major mergers, minor and very minor mergers, to those that have not had mergers, at fixed stellar mass. The medians in the right-hand panel show this trend more clearly. Interestingly, most galaxies, even main sequence galaxies, show $\delta_{\text {stars }}>0$, indicating $\sigma_{\|}>\sigma_{\perp}$. Thob et al. (2019) found that the most flattened systems are also the ones with the highest $\delta_{\text {stars }}$ due to the fact that in a flat system you expect little vertical stellar velocity dispersion, which leads to a smaller scale height. Major mergers therefore act to dynamically heat the galaxies making $\sigma_{\perp}$ approach $\sigma_{\|}$.

The third panels of Fig. 7 show the 3D Sèrsic index, $n_{\text {Sersic }}$ (measured from the 3D stellar mass distributions). There is a trend between $n_{\text {Sersic }}$ and the assembly history of an SR galaxy, whereby galaxies that have had major/minor mergers tend to have higher $n_{\text {Sersic }}$ than those that had only very minor mergers or no mergers at all.

${ }^{2}$ If our sample of interest is A and we want to draw a subsample from B to have the same stellar mass distribution of A, we randomly choose $N$ galaxies in narrow stellar mass bins from $\mathrm{B}$, where $N$ is the number of galaxies of that stellar mass in A. In our case A are the subsamples of SRs split by their merger history, and B are either all fast rotators or SRs in EAGLE.
Note that SRs with no mergers or very minor mergers have lower $n_{\text {Sersic }}$ than even main sequence galaxies. Lagos et al. (2018a) showed that in EAGLE, galaxies that have had dry or wet mergers had a higher $n_{\text {Sersic }}$ than galaxies without mergers. Here, we show that trends with merger history remain even when we select slow rotators only.

The fourth panels of Fig. 7 show that the ex-situ fraction strongly increases going from SRs without mergers, to SRs that have had $\geq 1$ major mergers, at fixed stellar mass. The subsample of SRs without mergers has an even smaller $f_{\text {ex-situ }}$ than main sequence galaxies of the same stellar mass, while the subsample of SRs with exclusively very minor mergers appears similar to main sequence galaxies. Interestingly, at $M_{\star} \gtrsim 10^{11} \mathrm{M}_{\odot}$, SRs that went through $N \geq 1$ major merger have as much $f_{\text {ex-situ }}$ as those that went only through minor mergers.

The fifth and bottom panels of Fig. 7 show a tendency for SRs without mergers to be more compact and have a lower BH-tostellar mass ratio than the rest of the SRs at fixed stellar mass. The half-light radius increases from SRs that exclusively had very minor mergers to those that had major or minor mergers. The latter are also the ones with the highest $\mathrm{BH}$-to-stellar mass ratio. These differences are suggestive of different quenching mechanisms between the subsamples. This is further discussed in the next section.

\subsection{Kinematic transformation and quenching of slow rotators}

The top panel of Fig. 8 shows the evolution of $\lambda_{\mathrm{r}_{50} \text {,edge-on }}$ of $z=0 \mathrm{SRs}$ classified by their merger history. We find that they follow similar $\lambda_{\mathrm{r}_{50} \text {, edge-on }}$ evolutionary tracks, in which most of the transformation happens in the last $6 \mathrm{Gyr}$, on average, and by $<2$ Gyr they are almost all completed. The main difference between SRs that went through different merger histories is when they formed their stars. SRs without mergers are the youngest ones, while those that went exclusively through very minor or minor mergers are the oldest, again suggesting different quenching mechanisms. The top panels of Fig. 9 shows this more clearly. The sSFR of SRs without mergers deviated from the main sequence at later times than other SRs, and by $z=0$ some of them continue to have low levels of star formation $(\approx 10-20$ times below the main sequence, on average $)$. On the other hand, the rest of the SRs are much more quenched by $z=0$, with those having only very minor mergers being the first to deviate from the main sequence at $\approx 9 \mathrm{Gyr}$ of look-back time. We find that in the sample of SRs without mergers, deviations from the main sequence are accompanied by changes in $\lambda_{\mathrm{r}_{50}}$, which start happening on average at a look-back time of 6 Gyr. This is not the case for the other SRs, where the kinematic transformation is disconnected from the star formation quenching. Even though the medians of the evolutionary tracks of $\lambda_{\mathrm{r}_{50}}$ are smooth, by visual inspection of individual tracks, we find that most galaxies tend to display a sharp decrease in $\lambda_{\mathrm{r}_{50}}$ (suggestive of mergers). The timing of galaxy mergers average out to give a smooth average track but leading to a large scatter around the median at look-back times $\gtrsim 1.5-2 \mathrm{Gyr}$; the quick transformation of $\lambda_{\mathrm{r}_{50}}$ in individual galaxies is happening mostly throughout look-back times $\approx 2-6$ Gyr. This agrees with the low fraction of SRs found in observations at $z \approx 0.6$, which increases rapidly to $z=0$ (Cole et al. 2020).

The bottom panels of Figs 8 and 9 focus on SRs that had $N \geq 1$ mergers (of any mass ratio), but we separate them between wet and dry mergers (based on whether $M_{\text {SFgas,total }} / M_{\star, \text { total }}$ is $>$ or $<0.1$, respectively; see Section 2.1 for details). SRs that had $N \geq 1$ dry mergers are older and have progenitors with higher $\lambda_{\mathrm{r}_{50} \text {,edge-on }}$ than the counterparts with $N \geq 1$ wet mergers and no dry mergers. This happens because dry mergers are more effective at decreasing 


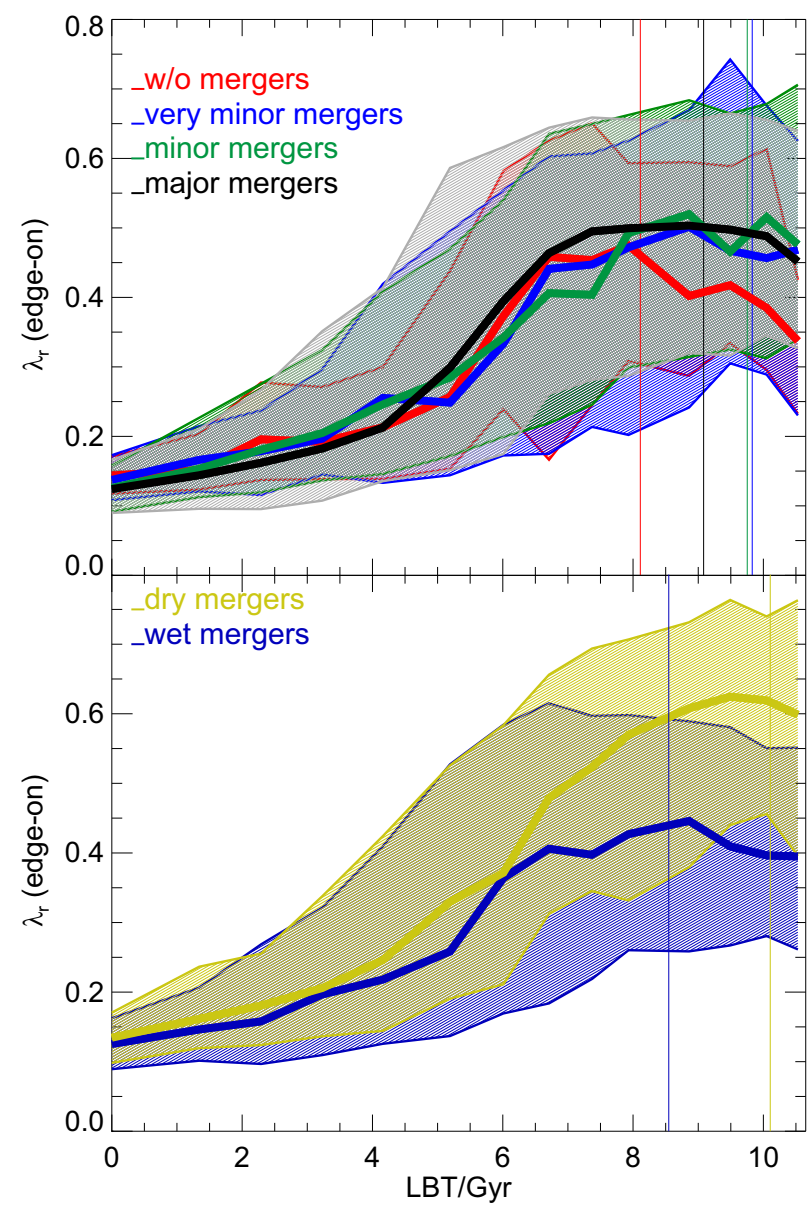

Figure 8. Top panel: The $\lambda_{\mathrm{r}_{50} \text {, edge-on }}$ (measured orienting galaxies edgeon) history of $z=0$ SRs that had $\geq 1$ major mergers, $=0$ major but $\geq 1$ minor mergers, $=0$ major/minor mergers but $\geq 1$ very minor mergers, and $=0$ mergers in the last $10 \mathrm{Gyr}$, as labelled. Lines with shaded regions show the median and 25 th -75 th percentile range, respectively. The vertical lines show the median $r$-band weighted stellar age of the samples. The latter was computed with all stellar particles within $r_{50}$ at $z=0$. Bottom panel: as in the top panel but for SRs that had $\geq 1$ wet or dry merger (of any mass ratio) in the last $10 \mathrm{Gyr}$, as labelled.

$\lambda_{\mathrm{r}_{50} \text {,edge-on }}$, as shown by Lagos et al. (2018a). Although by $z=0$ both types of SRs have similarly low sSFRs, SRs that had $N \geq 1$ dry merger started deviating from the main sequence earlier than those that had $N \geq 1$ wet merger, explaining their older age.

One key question is how these SRs quenched - or similarly, what led them to start deviating from the main sequence. Because of the high stellar masses of these galaxies $\left(\geq 10^{10} \mathrm{M}_{\odot}\right)$, there are only two plausible pathways in which they could have quenched in EAGLE: due to AGN feedback or environmental effects. The latter mostly happens in EAGLE due to tidal interactions between galaxies, tidal stripping, and ram pressure stripping (Marasco et al. 2016; Bahé et al. 2017). We explore this by separating the merger samples of SRs of Fig. 9 into centrals and satellites. Fig. 10 shows the fraction of galaxies among SRs that had different merger histories that are satellites by $z=0$. SRs that have not had mergers or have had exclusively very minor mergers have a clear preference for being satellite galaxies compared to other slow and fast rotators of the same stellar mass. Within SRs, those that have had major or minor mergers make the vast majority of central galaxies. These results already indicate environment has likely played an important role in

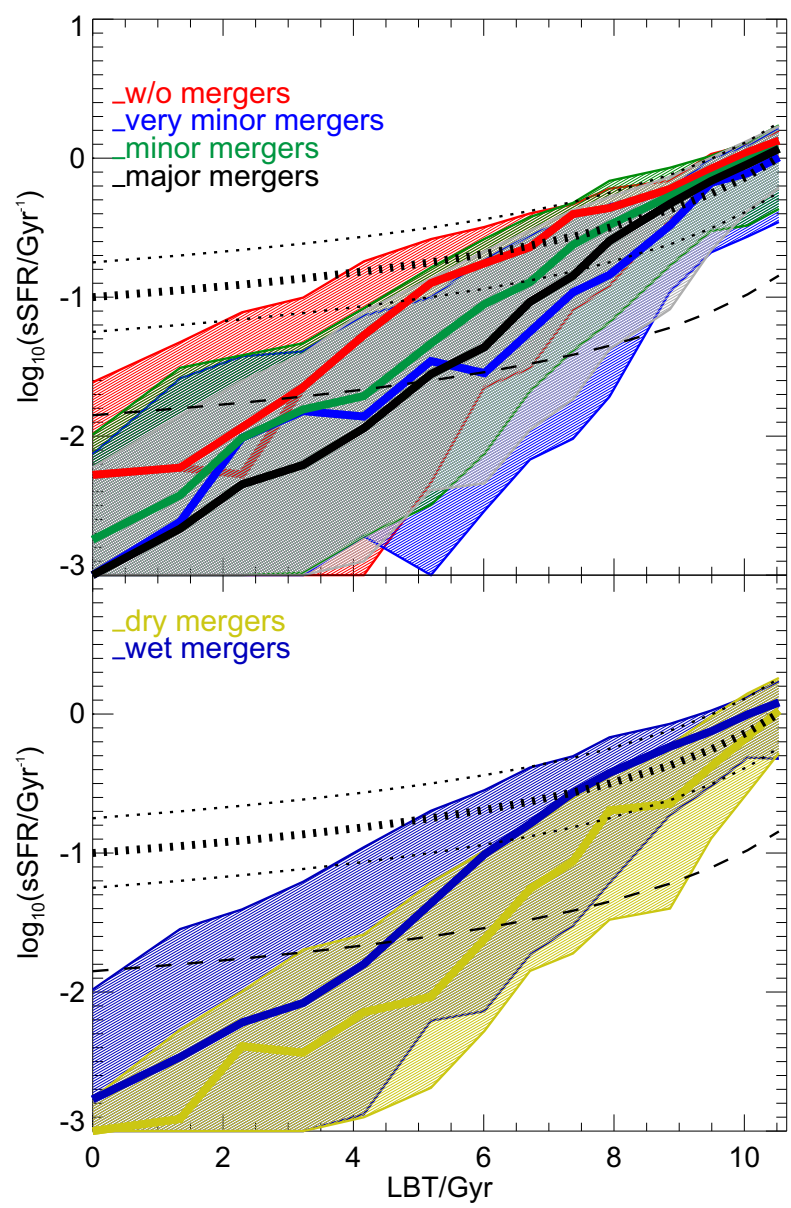

Figure 9. As in Fig. 8 but for the sSFR history. Here, the thick and thin dotted lines show the position of the main sequence in EAGLE at $M_{\star} \approx 10^{10} \mathrm{M}_{\odot}$, and a scatter of \pm 0.25 dex, respectively, which is approximately the value measured by Furlong et al. (2015) in EAGLE. The dashed line shows a distance to the main sequence of $-0.85 \mathrm{dex}$, which we use to define quenched galaxies.

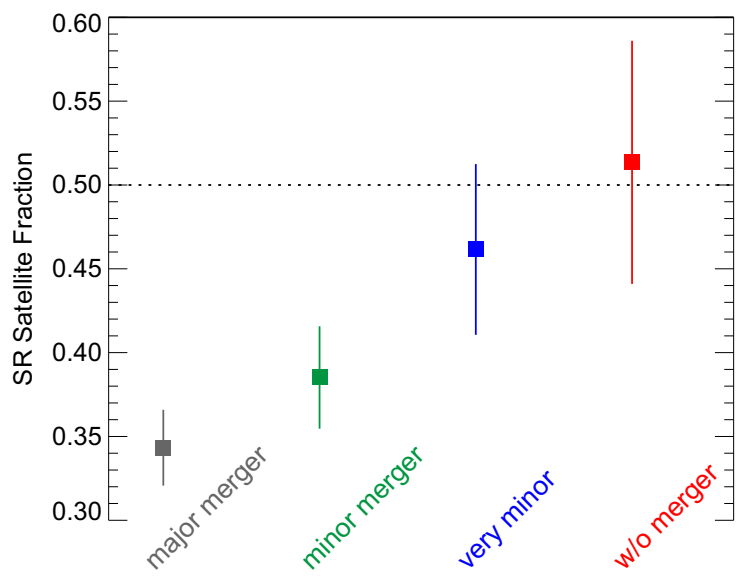

Figure 10. The fraction of $z=0$ galaxies that are classed as satellites, for SRs that had $\geq 1$ major mergers (grey shaded region), no major mergers but $\geq 1$ minor mergers (green), no minor or major mergers, but $\geq 1$ very minor mergers (blue) and no mergers (red) over the last $10 \mathrm{Gyr}$ (solid lines). Errorbars show Poisson errors. SRs that had exclusively very minor mergers or no mergers have a much higher probability of being a satellite galaxy than other SRs. 


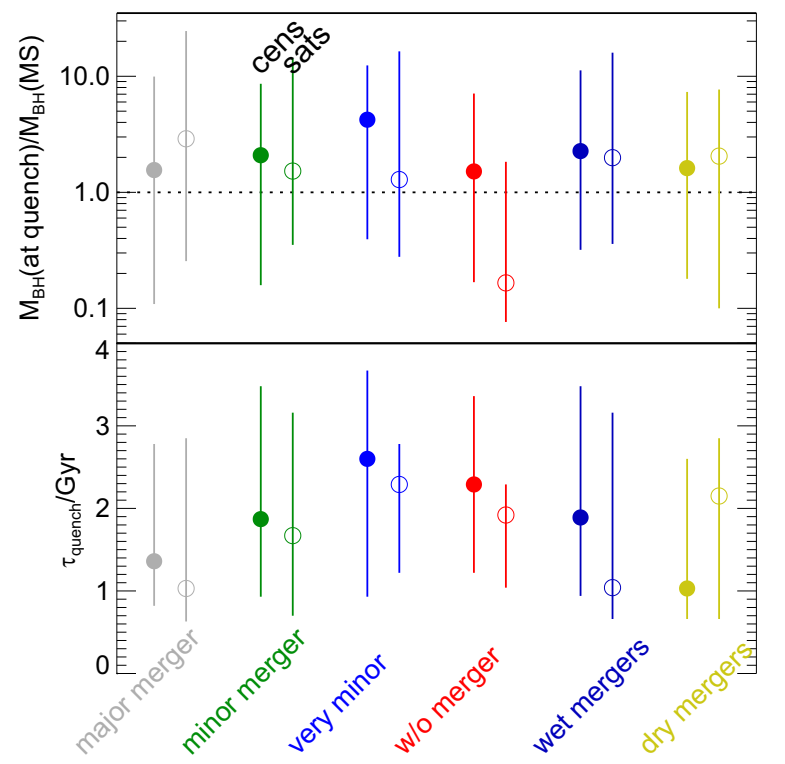

Figure 11. Top panel: The ratio between the BH mass of the SRs in Fig. 9 and the median $\mathrm{BH}$ mass of main sequence galaxies of the same stellar mass at the time the SRs leave the main sequence for the first time (lower dotted line in Fig. 9). We show this for central (filled circles) and satellite (open circles) galaxies separately. The symbols with error bars show the median and 25 th -75 th percentile range, respectively. Ratios $\geq 1$ correspond to galaxies that leave the main sequence with an overly massive $\mathrm{BH}$ compared to galaxies on the main sequence of the same stellar mass. Bottom panel: Quenching time-scale of the SRs in the top panel, defined as the time galaxies take to transition from the lower dotted to the dashed lines in Fig. 9.

quenching SRs mostly for the subsamples that had no mergers or exclusively very minor mergers. However, the excess in satellites does not uniquely point to environment as a source of quenching.

To isolate environment from AGN feedback as potential sources of quenching, we track back the time at which each SR departed the main sequence for the first time ( $\tau_{\text {depart }}$; which corresponds to the first time the sSFR of the SRs crossed the lower dotted line in Fig. 9) and measure their central BH mass, $M_{\mathrm{BH} \text { (at quench). Bower }}$ et al. (2017) showed that in EAGLE, galaxies being quenched by AGN feedback are characterized by a strongly non-linear BH growth phase, which makes the relative $\mathrm{BH}$-to-stellar mass ratio a good indicator of AGN feedback in action. We normalize $M_{\mathrm{BH} \text { (atquench) }}$ by the median central $\mathrm{BH}$ mass of main sequence galaxies of the same stellar mass of the SR's progenitor at $\tau_{\text {depart }}, M_{\mathrm{BH}(\mathrm{MS})}$, and save the ratio, $M_{\mathrm{BH}(\text { at quench) }} / M_{\mathrm{BH}(\mathrm{MS})}$. The top panel of Fig. 11 shows the median and 25th-75th percentiles of the distribution of $M_{\mathrm{BH} \text { (at quench) }} / M_{\mathrm{BH}(\mathrm{MS})}$ for $z=0$ SRs selected based on their merger histories. We show this separately for SRs that by $z=0$ are centrals and satellites. Overall we see a tendency for SRs to have overly massive BHs compared to main sequence galaxies of the same stellar mass at $\tau_{\text {depart }}$. The only exceptions are SRs that have not had mergers and end up as satellite galaxies by $z=0$; this population has light black holes compared to main sequence galaxies at $\tau_{\text {depart }}$.

Trayford et al. (2016) showed that excess BH mass is a strong indicator of colour transformation and quenching; Trayford et al. (2016), Wright et al. (2019) quantified that and showed that galaxies with overly massive $\mathrm{BHs}$ or high specific $\mathrm{BH}$ growth rates quench much more rapidly than those with lighter BHs (relative to their stellar mass), both in terms of colour transformation, as well as departures from the sSFR main sequence. Hence, the top panel of Fig. 11 suggests that the vast majority of SRs quenched due to AGN feedback, with the exception of $z=0$ satellite SRs that have not had mergers. We also highlight that AGN are likely to be the source of quenching even for satellite SRs that have had major, minor, or very minor mergers. We caution that this interpretation of overly sized BHs being an indicator of AGN feedback quenching applies to EAGLE (see Bower et al. 2017). However, this may not work in simulations implementing different models of AGN feedback that are not tied to rapid $\mathrm{BH}$ growth phases. Note that the trends of $M_{\mathrm{BH}(\text { atquench) }} / M_{\mathrm{BH}(\mathrm{MS})}$ at $\tau_{\text {depart }}$ for these different SRs continues to hold at later times, as shown in the bottom panels of Fig. 7 for $z=0$. The top panel of Fig. 11 also shows $M_{\mathrm{BH} \text { (atquench) }} / M_{\mathrm{BH}(\mathrm{MS})}$ at $\tau_{\text {depart }}$ for $z=0$ SRs that had $\geq 1$ dry or wet merger in the last $10 \mathrm{Gyr}$. Overall these are similar to the overall major/minor merger SRs subsample.

The bottom panel of Fig. 11 shows the quenching time-scale of these SRs defined as the time it took to transition from the main sequence down to an arbitrary low level of star formation, $\tau_{\text {quench }}$. In this paper, we use the method of Wright et al. (2019), which consists of measuring the time it took for a galaxy to change its sSFR from $\operatorname{MS}\left(M_{\star}\right)-c_{\text {high }}$ to $\operatorname{MS}\left(M_{\star}\right)-c_{\text {low }}$, where $\operatorname{MS}\left(M_{\star}\right)=$ $\log _{10}\left(\mathrm{sSFR}_{\mathrm{MS}}\left(\mathrm{M}_{\star}\right) / \mathrm{Gyr}^{-1}\right)$ is the sSFR of the main sequence at $M_{\star}$. Here, we adopt $c_{\text {high }}=0.25$ and $c_{\text {low }}=0.85$ (lower thin dotted and dashed lines in Fig. 9). Note that these values are slightly different to those adopted in Wright et al. (2019), but they give us the best statistics for $\tau_{\text {quench }}$, as the value of $c_{\text {low }}=1.3$ dex adopted in Wright et al. (2019) leads to about half of the SRs in the 'no merger' subsample to have undefined $\tau_{\text {quench. }}$. We note that typical values for $c_{\text {low }}$ adopted in the literature range from $\approx 1.6$ to 0.3 dex (Béthermin et al. 2015; Davies et al. 2018; Wang et al. 2018).

Most SRs have $\tau_{\text {quench }} \approx 1-2 \mathrm{Gyr}$, in agreement with quenching time-scales derived in observations (Smethurst et al. 2018). Note that satellite SRs in the 'no merger' sample quench fast, $\tau_{\text {quench }} \approx$ 1.9 Gyr, despite them having overly light BHs. This is the only subsample in which this happens. If we focus on central galaxies that by $z=0$ are SRs and had galaxy mergers, we find that changes in $\lambda_{\mathrm{r}_{50} \text {,edge-on }}$ happen after galaxies quench, on average (galaxies start deviating from the main sequence earlier than they start showing $\left.\lambda_{\mathrm{r}_{50} \text {,edge-on }} \lesssim 0.5\right)$. We quantify this by comparing the look-back time at which the SRs' progenitors leave the main sequence $\left(c_{\text {high }}=0.25\right)$ with the time at which progenitors have a $\lambda_{\mathrm{r}_{50} \text {,edge-on }}$ that is 0.8 times the maximum $\lambda_{\mathrm{r}_{50}, \text { edge-on }}$ they had. We find that for SRs that have had mergers, quenching starts happening $\approx 1-2$ Gyr before $\lambda_{\mathrm{r}_{50} \text {,edge }- \text { on }}$ drops below 80 percent its maximum historical value, while for SRs that have not had mergers, this happens roughly at the same time (within $0.2 \mathrm{Gyr}$ ). The emerging picture is that AGN feedback quenches these centrals galaxies at $z \gtrsim 1$, and subsequent mergers are responsible for the kinematic transformation leading them to become SRs.

To address the effect of environment in quenching and potential transformation of the kinematics of satellite galaxies, we show in the left-hand panel of Fig. 12 the relative change of $\lambda_{\mathrm{r}_{50} \text {,edge-on }}$, $\delta \lambda_{\mathrm{r}_{50} \text {,edge-on }}$, and sSFR, $\delta \mathrm{sSFR}$, for satellite SRs in our four samples split by their merger history. This relative change is computed between $z=0$ and the last time the galaxy was a central,

$\delta i=\frac{i(z=0)-i(\text { last central })}{i(\text { last central })}$,

with $i=\lambda_{\mathrm{r}_{0} \text {,edge-on }}$ or sSFR. By definition, $\delta i \geq-1$, with a value $\delta i=$ -1 indicating the quantity of interest at $z=0$ is $=0$ (which is often the case for SSFR). The look-back time to when satellite SRs were last central has a median of $\approx 6 \mathrm{Gyr}$, and a 16 th and 84 th percentiles of 

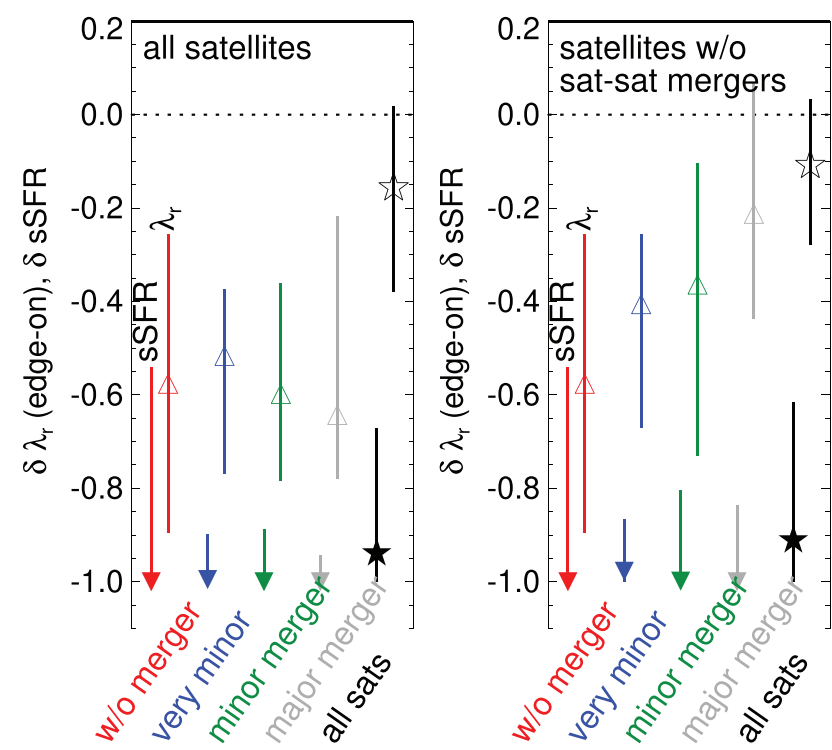

Figure 12. Left-hand panel: Relative change in $\lambda_{r_{50}}$ (edge - on) (up-pointing triangles) and sSFR (down-pointing triangles) for the subsample of satellite $z=0$ SRs of Fig. 10, between $z=0$ and the time they were last central. Symbols with error bars show the median and 25 th -75 th percentile range. We also show for reference the relative change in $\lambda_{r_{50}}($ edge - on) and sSFR for all $z=0$ satellite galaxies in EAGLE as black stars. Right-hand panel: As in the left-hand panel but only for satellites that have not had a galaxy merger since becoming a satellite (i.e. no satellite-satellite mergers).

$\approx 2.5 \mathrm{Gyr}$ and $\approx 8.6 \mathrm{Gyr}$, respectively (most of them became satellites at $z<1$, as expected). The right-hand panel of Fig. 12 also shows $\delta \lambda_{\mathrm{r}_{0} \text {,edge-on }}$ and $\delta \mathrm{sSFR}$ for the whole population of $z=0$ satellite galaxies with $M_{\star} \geq 10^{10} \mathrm{M}_{\odot}$. We find that in all cases, satellites that by $z=0$ are visually classified as SRs suffered significant quenching and kinematic transformation since becoming a satellite galaxy. The case of the whole population of satellites is very different; quenching here is unaccompanied by kinematic transformations. Even though most satellites suffer an overall decrease in $\lambda_{\mathrm{r}_{50} \text {, edge-on }}$, this is small compared to the change in SSFR. The latter is consistent with what Cortese et al. (2019) inferred for satellite galaxies in the SAMI survey, and agree with the analysis presented there for EAGLE in which net changes in stellar rotation-to-dispersion velocity ratio were compared to net changes in SFR to find that the two were decoupled. We investigate the effect of environment further by studying how many of these $z=0$ SR satellites suffered their last merger after becoming satellites. We find that this happens frequently: $\approx 50$ percent of SR satellites had their last merger after becoming satellites (for the general population of satellites with $M_{\star} \geq 10^{10} \mathrm{M}_{\odot}$ this is much lower, $\approx 20$ per cent). To isolate the environment effect (which we associate with interactions with the tidal field of the halo and the central galaxy) from those of mergers with other satellite galaxies, the right-hand panel of Fig. 12 shows the subsample of satellites that had their last merger prior to becoming satellites. We see significant differences with the left-hand panel of Fig. 12. Overall, the relative change in $\lambda_{\mathrm{r}_{50} \text {,edge-on }}$ is significantly smaller for satellites that had mergers (particularly major or minor mergers) prior to becoming satellites. This shows that satellite-satellite mergers are at least as effective (or more so) in reducing $\lambda_{\mathrm{r}}$ as the environment (as defined here). In the sample of SRs of the right-hand panel of Fig. 12, there is a trend of the kinematic transformation being weaker when going from SRs that had exclusively very minor, minor to major mergers prior to becoming satellites. The weak environmental effect on $\lambda_{\mathrm{r}_{0}, \text { edge-on }}$ in SRs that have had mergers is due to the fact that by the time they become satellites they already have low $\lambda_{\mathrm{r}_{50} \text {,edge-on }} \approx 0.19-0.29$, with the lower (higher) value corresponding to the median for SRs at the time of accretion that had major (only very minor) mergers prior to becoming satellites. In comparison, satellites that have not had mergers by $z=0$ and are SRs had a median $\lambda_{\mathrm{r}_{50} \text {,edge-on }} \approx 0.47$ by the time they became satellites. For reference, satellite fast rotators at $z=0$ had a median $\lambda_{\mathrm{r}_{50} \text {,edge-on }} \approx 0.6$ at the time they became satellites.

In contrast, SRs that had mergers after becoming satellites were accreted with much higher $\lambda_{\mathrm{r}_{50} \text {, edge-on }} \approx 0.5$, on average, showing that those that do not experience mergers during their lifetime as satellites suffer from strong progenitor bias. ${ }^{3}$ The comparison between the left-hand and right-hand panels of Fig. 12 also shows that the large kinematic transformation seen in the left-hand panel for SRs that have had mergers is driven in great part by satellitesatellite mergers (particularly for those that go through minor/major mergers with other satellites) rather than by interactions with the central galaxy or the tidal field of the host halo (which are the main mechanisms of kinematic transformation due to the environment; Choi \& Yi 2017).

Choi \& Yi (2017) analysed satellite SRs in the Horizon-AGN hydrodynamical simulations and found that only 22 per cent of their satellite SRs appeared to have low spins due to galaxy mergers. This appears to be in contradiction with our findings in EAGLE. Part of that can be related to the high contamination parametric selections used in Choi \& Yi (2017) have in distinguishing unambiguous SRs (see Section 2.4), but more likely is that there are significant differences in the properties of satellite galaxies between the two simulations that allow environment to play a more significant role in the spindown of galaxies in Horizon-AGN compared to EAGLE.

Despite the clear trends found in EAGLE between the properties of SRs and their assembly history, it is important to highlight that having had mergers of some sort does not guarantee the formation of an SR. In fact, many fast rotators have also gone through galaxy mergers of different mass ratios and gas content (as seen from the difference in the number of visually classified SRs, 479, and the number of galaxies that went through different merger histories in Table 2). The emerging picture from EAGLE is that the required condition to form an SR is the process of quenching prior to or simultaneously with the kinematic transformation.

\subsection{The stellar populations of slow rotators}

The different star formation and assembly histories of SRs in EAGLE should leave imprints on the stellar populations of these galaxies that are potentially observable. Here, we focus on the metal abundance and stellar ages of $z=0$ SRs in EAGLE.

Fig. 13 shows the radial profile of the abundance of $\alpha$ elements relative to Fe. We compute $[\alpha / \mathrm{Fe}]=\log _{10}\left(\mathrm{M}_{\alpha} / \mathrm{M}_{\mathrm{Fe}}\right)-\log _{10}(\alpha / \mathrm{Fe})_{\odot}$, where $M_{\alpha}$ is the mass contributed by $\alpha$ elements (the sum of the masses contained in $\mathrm{Si}, \mathrm{O}, \mathrm{Mg}, \mathrm{Ne}$, and $\mathrm{C}$ ), $M_{\mathrm{Fe}}$ the mass in iron and $\log _{10}(\alpha / \mathrm{Fe})_{\odot}=13.1206$ (Asplund, Grevesse \& Sauval 2005). The top panel of Fig. 13 shows $z=0$ SRs split by their assembly history, as labelled; also shown is the median $[\alpha / \mathrm{Fe}]$ of main sequence galaxies ( $\mathrm{sSFR}>0.01 \mathrm{Gyr}^{-1}$ ) with $M_{\star}>10^{10} \mathrm{M}_{\odot}$. All SRs that have had mergers are $\alpha$-enhanced relative to the sun across the whole radial

\footnotetext{
${ }^{3}$ Their progenitors have sufficiently different properties as to cause the differences seen at $z=0$ with the SRs that had satellite-satellite mergers.
} 


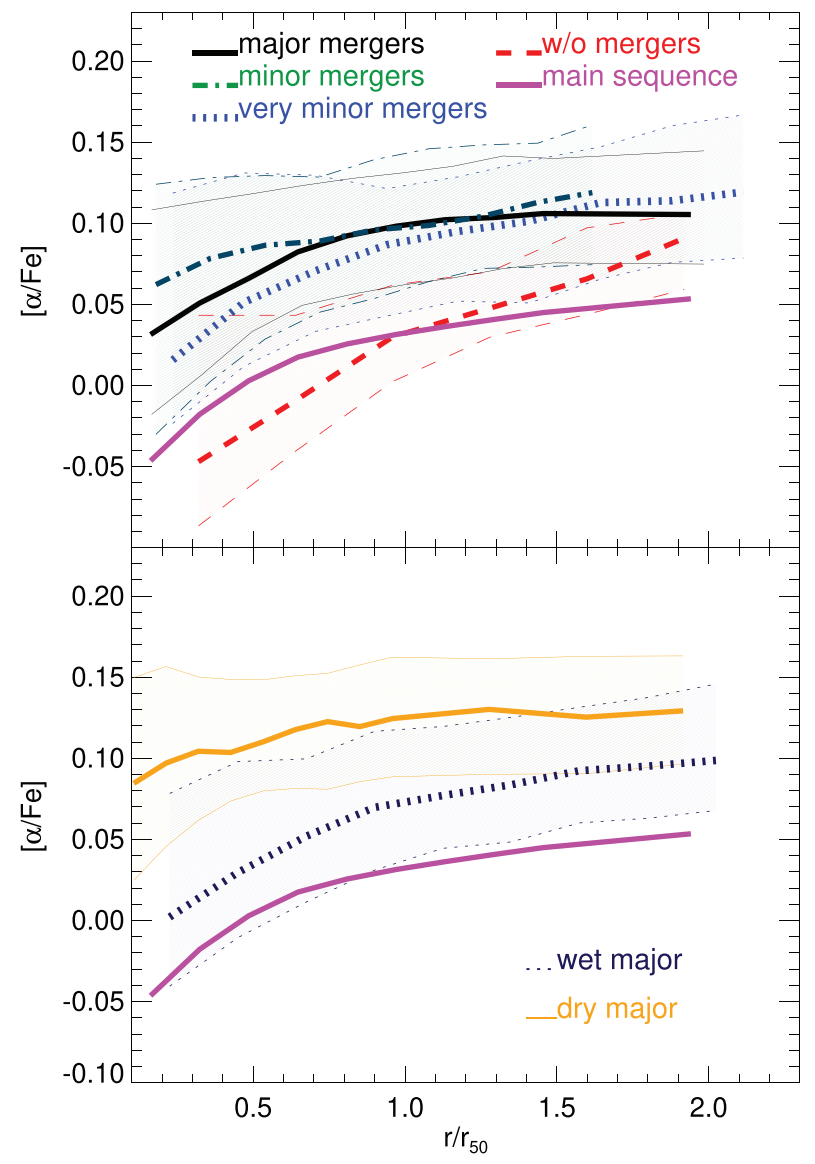

Figure 13. Top panel: Radial profiles of the stellar abundance of $\alpha$-elements over iron, in units of the solar abundance, $[\alpha / \mathrm{Fe}]$, for SRs at $z=0$ that have had $\geq 1$ major mergers (solid lines), $=0$ major but $\geq 1$ minor mergers (dot-dashed lines), $=0$ major/minor mergers but $\geq 1$ very minor mergers (dotted lines), and $=0$ mergers (dashed lines) in the last 10 Gyr. The thick lines show the median and the shaded regions plus thin lines show the 25 th -75 th percentile range, respectively. The magenta lines show the median for galaxies with $M_{\star}>10^{10} \mathrm{M}_{\odot}$ and sSFR $>0.01 \mathrm{Gyr}^{-1}$ (considered to be representative of the main sequence). Radii are normalized by the half $r$-band luminosity radii of galaxies. Bottom panel: as in the top panel but for SRs that had $\geq 1$ wet (dotted line) or dry (solid line) merger (of any mass ratio) in the last $10 \mathrm{Gyr}$, as labelled.

range investigated. This is not the case for SRs that have not had mergers, in which $[\alpha / \mathrm{Fe}]<0$ at $r<r_{50}$, on average $\approx 0.1$ dex lower than $[\alpha / \mathrm{Fe}]$ of other SRs. Interestingly, SRs in the 'no merger' group are even less $\alpha$-enhanced than main sequence galaxies at $r<r_{50}$. This shows that low $[\alpha / \mathrm{Fe}] \mathrm{SRs}$ are more likely to belong to the "no merger' sample than the other ones. The bottom panel of Fig. 13 shows $[\alpha / \mathrm{Fe}]$ radial profiles this time for SRs that had wet or dry mergers of any mass ratio (see Section 2.1). Those that had dry mergers are the ones with the flattest and most $\alpha$-enhanced $[\alpha / \mathrm{Fe}]$ radial profiles.

A general feature is that most galaxies in EAGLE tend to exhibit inverted $[\alpha / \mathrm{Fe}]$ profiles in which the central parts are less $\alpha$ enhanced than the outer parts. This happens because, on average, the stellar age radial profiles are inverted in EAGLE, with the central parts being younger than the outer parts. Although early-type and passive galaxies in observations are consistent with flat (or even inverted) stellar age and $[\alpha / \mathrm{Fe}]$ radial profiles (e.g. Kuntschner et al. 2010; Greene et al. 2015; Li et al. 2018a; Bernardi et al. 2019;
Barsanti et al. 2020; Santucci et al. 2020), late-type galaxies tend to have stellar age profiles consistent with the inner parts being older (e.g. González Delgado et al. 2015; Barsanti et al. 2020). For starforming galaxies with $M_{\star}>10^{10} \mathrm{M}_{\odot}$ and sSFR $>0.01 \mathrm{Gyr}^{-1}$ at $z=$ 0 in EAGLE, we find $\approx 88$ per cent have inverted stellar age profiles (younger central parts; a similar percentage is found for those with sSFR $<0.01 \mathrm{Gyr}^{-1}$ ), which disagrees with observational evidence. We do note, however, that the integrated $[\alpha / \mathrm{Fe}]$ ratios in EAGLE galaxies agrees well with observations (Segers et al. 2016). This shows that even though feedback in EAGLE is sufficient to quench star formation to reproduce the correct stellar mass function and other global properties related to metallicities and element abundances, the predicted radial properties of the stellar populations in galaxies has some important discrepancies with observations. The excess star formation in the centre is then the likely culprit of many of EAGLE galaxies exhibiting inverted stellar velocity dispersion radial profiles, where the central velocity dispersion is lower (see for example the top three and bottom panels of Fig. 1). Section 2.2 reported that $\approx 55$ percent of galaxies with $M_{\star}>10^{10} \mathrm{M}_{\odot}$ have $\sigma_{\star}\left(0.5 \mathrm{r}_{50}\right)<\sigma_{\star}\left(\mathrm{r}_{50}\right)$. This percentage reduces to 43 percent for passive galaxies or SRs in EAGLE. This is much larger than what is reported in observations. Falcón-Barroso et al. (2017) found that in the sample of early-type galaxies in CALIFA, only 1 or 2 galaxies (out of 47) have $\sigma_{\star}\left(0.5 \mathrm{r}_{50}\right)<\sigma_{\star}\left(\mathrm{r}_{50}\right)$.

In the galaxies with $\sigma_{\star}\left(0.5 \mathrm{r}_{50}\right)<\sigma_{\star}\left(\mathrm{r}_{50}\right)$, the $r$-band weighted stellar ages increase from $7.7 \mathrm{Gyr}$ at $r<0.5 r_{50}$ to $8.2 \mathrm{Gyr}$ at $r<r_{50}$, on average, showing the connection between the lower central stellar velocity dispersions and the inverted stellar age profiles. We measure the Pearson correlation coefficient between $\log _{10}\left(\sigma_{\star}\left(0.5 \mathrm{r}_{50}\right) / \sigma_{\star}\left(\mathrm{r}_{50}\right)\right)$ and $\log _{10}\left(\operatorname{age}_{\star}\left(0.5 \mathrm{r}_{50}\right) / \operatorname{age}_{\star}\left(\mathrm{r}_{50}\right)\right)$, for all galaxies with $M_{\star}>10^{10} \mathrm{M}_{\odot}$ and obtained $P=0.43$. This correlation becomes stronger, $P=0.6$, for SRs in EAGLE, which shows that the more strongly inverted the stellar age profile, the more strongly inverted the $\sigma_{\star}$ profile. This is an important shortcoming of EAGLE that upcoming hydrodynamical simulations need to address.

Fig. 14 shows radial profiles of $[\mathrm{Fe} / \mathrm{H}]=\log _{10}\left(\mathrm{M}_{\mathrm{Fe}} / \mathrm{M}_{\mathrm{H}}\right)-$ $\log _{10}(\mathrm{Fe} / \mathrm{H})_{\odot}$ for $z=0$ SRs in EAGLE, with $(\mathrm{Fe} / \mathrm{H})_{\odot}=0.001798$ (Asplund et al. 2005). SRs in the 'no merger' sample have the highest metallicities due to their delayed quenching times compared to other SRs (see Fig. 9). Again, we see that these SRs have even higher $[\mathrm{Fe} / \mathrm{H}]$ than main sequence galaxies, and display the steepest radial profiles. SRs in the 'very minor merger' sample have the lowest and flattest $[\mathrm{Fe} / \mathrm{H}]$ radial profiles due to their early quenching (see Fig. 9). The bottom panel of Fig. 14 separates SRs between dry and wet mergers. Dry mergers lead to SRs that have flatter $[\mathrm{Fe} / \mathrm{H}]$ profiles due to the effective redistribution of stellar mass during dry mergers (Lagos et al. 2018b). Krajnović et al. (2020) found that classical slow rotators (which they linked to the dissipation-less galaxy mergers; i.e. dry mergers) have flatter metallicity gradients than other slow rotators in ATLAS ${ }^{3 \mathrm{D}}$, which is in qualitative agreement to what we find in EAGLE.

The trends shown in Figs 13 and 14 show that observations of the stellar populations in SRs can provide a broad indication of the most likely merger history. However, as these are trends, application on a one-to-one basis is not advised.

\section{KINEMATIC CLASSES OF SLOW ROTATORS}

In this section, we use the visual kinematic classification of EAGLE galaxies of Section 2.3 and analyse their connection to the galaxy merger history to understand the effect the galaxy mass ratio and gas ratio involved in mergers have on the kinematic class. We focus 


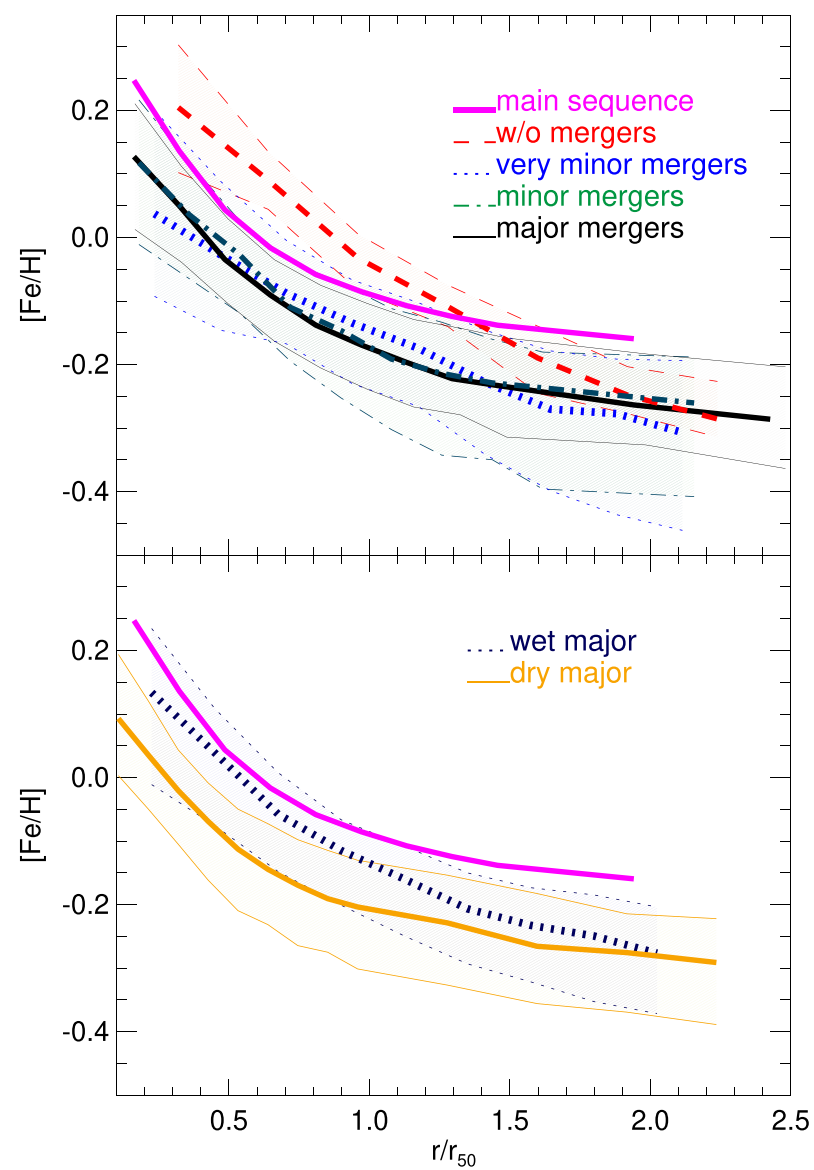

Figure 14. As in Fig. 13 but for radial profiles of $[\mathrm{Fe} / \mathrm{H}]$.

on the latter two merger parameters as Lagos et al. (2018a,b) have shown that those have the most effect in modifying the kinematics of galaxies. We also study possible connections between environment and stellar mass with the different SR's kinematic classes. In this section, we only study the kinematic classes FSR, RSR, and prolates (overall classed as SRs), ignoring the $2 \sigma$, unclear and rotator classes.

\subsection{The relation between kinematic class and galaxy merger history}

Fig. 15 shows the PDF of the SRs kinematic classes described in Section 2.3 for all the galaxies visually classified that have a confidence $\geq 60$ per cent, split by their merger history. By comparing galaxies that had $\geq 1$ major mergers and those that had $\geq 1$ minor mergers but no major mergers (left-hand panel), we see that the former tend to be associated with more FSRs, while prolate galaxies appear to have a preference for minor mergers. The overall distribution of galaxies that had exclusively very minor mergers is qualitatively similar to those that had no mergers (middle panel). The distribution of minor and very minor mergers are similar, and we see that a similar fraction of those are associated with FSRs and RSRs. Although prolates do happen in these SRs, their relative fraction is small compared to what is seen for SRs that had major/minor mergers.

The right-hand panel of Fig. 15 shows dry and wet mergers (see Section 2.1 for the criterion to define wet and dry mergers). We remind the reader that the sample of dry mergers can also contain wet mergers, while the sample of wet mergers excludes dry mergers.
We find that FSRs are overly represented in the sample of wet mergers compared to the dry merger sample. If we split wet mergers in two bins of gas fraction we obtain similar distributions (not shown). Although about half of the prolate galaxies formed via wet mergers and the other half via dry mergers in bulk numbers, we see that the normalized distributions of dry mergers have a higher incidence of prolate galaxies than wet mergers.

Fig. 16 shows the relative frequency of different types of galaxy mergers for a given kinematic class. We define this relative frequency as $=N_{\text {mer }}^{\text {sr class }} /\left(N_{\text {mer }}^{\text {all sr }} \times N_{\text {sr class }}\right)$, where $N_{\text {mer }}^{\text {sr class }}, N^{\text {all sr }}$, and $N_{\text {sr class }}$ are the number of SRs in a given kinematic class that went through the corresponding type of merger, the total number of SRs that went through that same type of merger, and the number of SRs in the kinematic class, respectively. This way we normalize by the different number of galaxies in each kinematic class and in each merger history type. We confirm that FSRs have a preference for major mergers compared to minor ones, while RSRs appear to be similarly represented for the minor, very minor, and no merger cases (considering the relative numbers of those). Prolate galaxies have a preference for dry mergers, and are also overly represented in the case of minor mergers. However, we caution that the latter is highly uncertain due to the small number of prolate galaxies in our sample (see Table 3).

In order to get a better understanding of the connection between the merger parameters and the different kinematic classes of SRs, we study the distribution of the stellar mass and gas ratios, and look-back time of the last galaxy merger each SR in our sample had (in this case we remove the sample of SRs that have not had mergers). This is shown in Fig. 17. Below we discuss the main trends in the three quantities shown in Fig. 17:

(i) Merger mass ratio. Comparing FSRs and RSRs, we see a preference of RSRs for smaller stellar mass ratios, even within the major merger band $\left(M_{\star, \sec } / M_{\star \text {, prim }}>0.3\right)$, compared to FSRs. FSRs are the sample that is most skewed towards high $M_{\star \text {, sec }} / M_{\star \text {, prim }}$. Prolate galaxies seem to be associated with either very low or intermediate mass ratios, $0.1 \lesssim M_{\star \text {, sec }} / M_{\star \text {, prim }} \lesssim 0.45$. We will show later that the lower stellar mass ratios are mostly associated with gas-poor mergers, while the higher ratios to gas-richer mergers.

(ii) Merger gas ratio. FSRs and RSRs show comparable distributions of gas ratios Prolates, on the other hand, prefer lower gas ratios compared to both FSRs and RSRs, that is most clear in the regime of dry mergers.

(iii) Look-back time to last merger. FSRs, RSRs, and prolates have a similar distribution of look-back time to their last galaxy mergers. Even though the errors are large there is a small preference for prolates to have had their last merger at later times. The latter would be expected given that gas poorer mergers happen preferentially at later times in EAGLE (Lagos et al. 2018b).

In the case of prolates, Li et al. (2018b) found that in the Illustris simulations they were predominantly associated with late, dry major mergers. In EAGLE we find a clear preference for dry mergers, but find that in bulk numbers a similar percentage of prolates are associated to major and minor mergers; i.e. $\approx 47$ percent to major mergers and $\approx 43$ percent to minor mergers. When the distributions are normalized by the relative numbers of these mergers in SRs, we find that minor mergers have a higher incidence of prolates. Furthermore, the remaining 10 per cent are associated to very minor mergers or no mergers. Hence, it appears like the formation mechanisms of prolates in EAGLE are more diverse than in Illustris.

To connect the stellar mass and gas ratios of the mergers of the different kinematic classes of SRs in EAGLE, we show in Fig. 18 the 

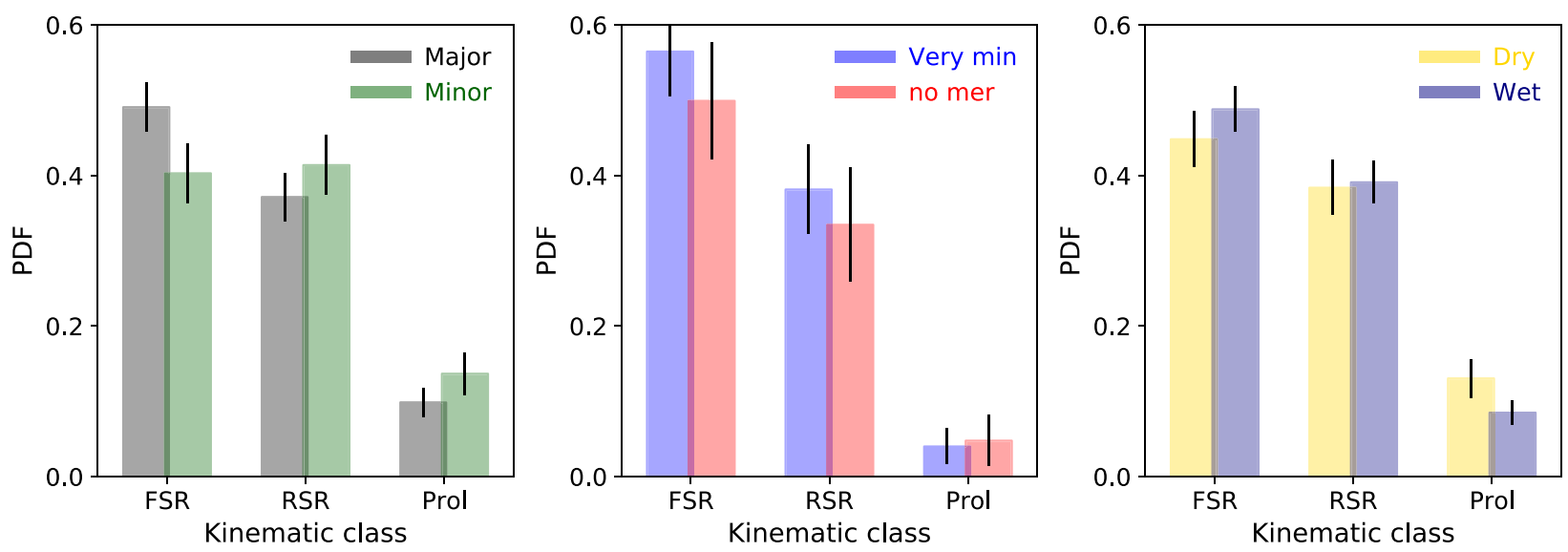

Figure 15. PDF of the kinematic classes of $z=0$ EAGLE galaxies in the visually classified sample split by their merger history. The left-hand panel shows those that had $\geq 1$ major mergers in the last $10 \mathrm{Gyr}$ and those that had $=0$ major mergers but $\geq 1$ minor mergers, as labelled. The middle panel shows those that had $=0$ major/minor mergers but $\geq 1$ very minor mergers and $=0$ mergers. The right-hand panel shows those that had $\geq 1$ dry mergers and those with $=0$ dry mergers but $\geq 1$ wet mergers. Error bars were computed from jackknife resampling and are displaced arbitrarily from the centre of the bin to aid visualization.

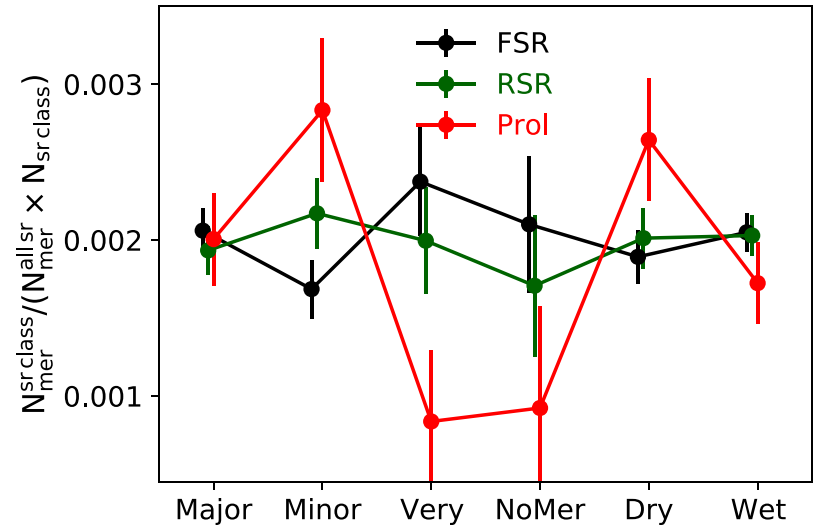

Figure 16. Relative frequency of different types of mergers for a given kinematic class. We show this for six different galaxy merger types: major mergers, minor mergers (with no major mergers), exclusively very minor mergers, no mergers, dry and wet mergers, counted over the last $10 \mathrm{Gyr}$ of evolution of these galaxies that at $z=0$ are classified as belonging to the four kinematic classes shown, as labelled. Error bars were computed from jackknife resampling.

distribution of the gas ratio of the last merger SRs went through, split into major and very minor plus minor mergers. Generally, we find that in all three kinematic SR classes, minor and very minor mergers that lead to remnant SRs tend to be gas poorer than major mergers leading to SRs. FSR and RSR show similar distributions of merger gas ratios in both panels, which means that the main difference between these two subclasses is the higher stellar mass ratios of the FSRs (Fig. 17). Prolates behave similarly, with the minor/very minor mergers being heavily skewed towards gas-poor mergers, and major mergers having a wider range of gas ratios. Connecting to the lefthand panel of Fig. 17, we find that for prolates, the lower (higher) stellar mass ratios are primarily associated with low (high) gas ratios.

Although the trends above are connected to possible physical drivers, it is important, however, to highlight that Poisson noise is quite significant in these trends due to low number statistics. Ideally we would like to study the 3D space between kinematic classes, stellar mass, and gas ratios but the current statistics in
EAGLE are prohibiting. We are then forced to marginalize over one of these properties. Upcoming simulations of much larger cosmological volume but comparable or even higher resolution than EAGLE are required to consolidate some of the trends reported here and to open the possibility to a much finer connection between merger parameters and the kinematic properties of SRs (including the extension to more mergers parameters associated with the orbits of satellite galaxies).

\subsection{The relation between kinematic class, stellar mass, and environment}

Fig. 19 shows the median stellar and halo masses of SRs in EAGLE split by their kinematic class. SRs of different kinematic classes have a similar median stellar masses, but prolate SRs tend to be skewed towards higher masses. The typical stellar masses of prolate galaxies in EAGLE agree well with those reported in Schulze et al. (2018) in the Magneticum simulations, but differ significantly from the ones in Illustris reported in Li et al. (2018b). Li et al. (2018b) found that prolates in Illustris are almost exclusively galaxies with $M_{\star} \gtrsim 3 \times 10^{11} \mathrm{M}_{\odot}$. Part of this discrepancy may come from the fact that at halo masses $>10^{12.2} \mathrm{M}_{\odot}$, Illustris produces galaxies too massive in stars compared to observational inferences by a factor of $\approx 7-10$ (see fig. 4 in Pillepich et al. 2018). EAGLE on the other hand produces a stellar-halo mass relation in better agreement with observations (Schaye et al. 2015). If one was to instead analyse the host halo masses of prolate galaxies in EAGLE and Illustris, the difference above would be largely alleviated.

For the halo masses of SRs in EAGLE (right-hand panel in Fig. 19), we find larger variations than for stellar mass. Interestingly, FSRs are more massive in stars but are hosted by lower mass haloes than RSRs, on average, and prolates have the highest stellar-to-halo mass ratio. This is the result of two factors: the fact that Fig. 19 includes both centrals and satellites (and for the latter we expect no correlation between stellar and host halo mass), and the fact that at fixed halo mass, the scatter in the stellar-halo mass relation is correlated with the assembly history of galaxies (e.g. Correa \& Schaye 2020). To disentangle these effects, we also show in Fig. 19 the median stellar and halo mass of central SRs only in the same three kinematic classes (squares). The most striking trend is that central prolates tend to have a slightly higher stellar-to-halo mass ratios (median 0.02) compared 

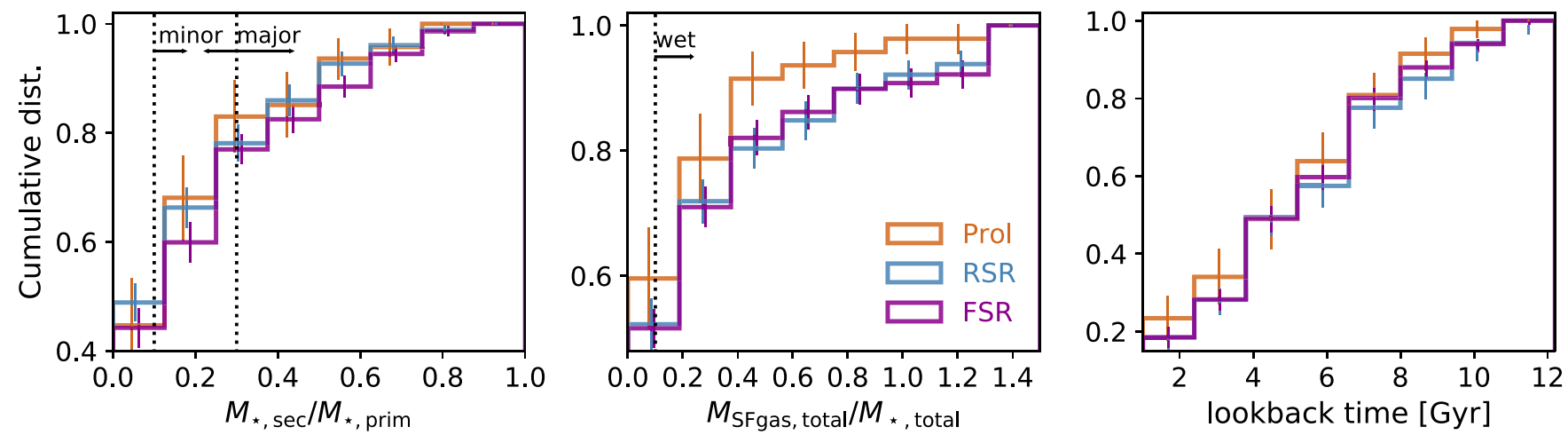

Figure 17. Cumulative distribution of the stellar mass ratio (left-hand panel), SF gas ratio (middle) and look-back time (right) of the last merger SRs in the four kinematic classes, labelled in the middle panels, went through. The vertical lines in the top and middle panels show what we classify as major/minor/very minor mergers, and wet/dry mergers, respectively. Error bars were computed from jackknife resampling and are displaced from the centre of the bin by arbitrary amounts to aid their visualization. Prolate SRs have a preference of lower gas ratios. Flat SRs prefer high stellar mass ratios compared to round SRs.
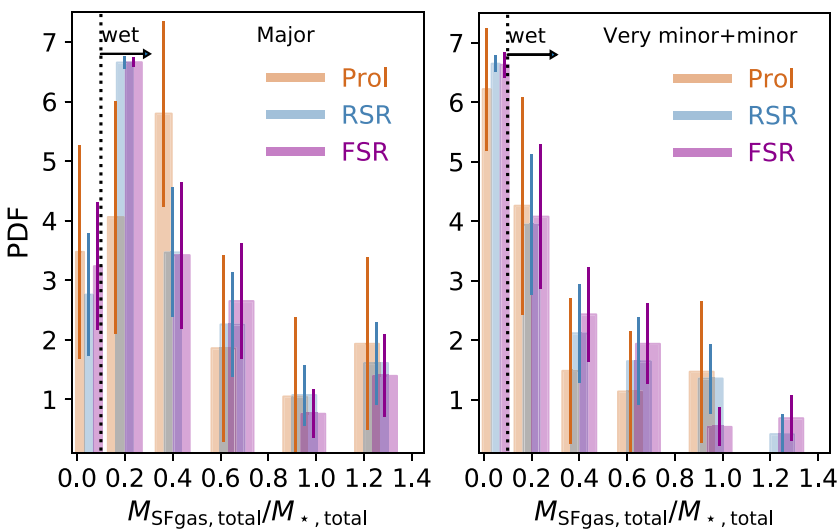

Figure 18. PDF of the SF gas ratio of the last major merger (left-hand panel) and very minor plus minor mergers (right-hand panel) SRs went through. We show this for 3 kinematic classes, as labelled. In the right-hand panel we show SRs that experienced very minor or minor mergers (no major mergers in their history), while the SRs in the left-hand panel has gone through major mergers. Error bars were computed from jackknife resampling and are displaced from the centre of the bin by arbitrary amounts to aid their visualization. Note that we use arbitrary bin widths that are narrower in the ranges where there are more galaxies. Very minor/minor mergers that lead to SRs are preferentially low gas ratios compared to the major mergers that lead to SRs.

to the other SRs by $\approx 12$ per cent. Given how tight the stellar-halo mass relation is in EAGLE (median stellar-to-halo mass ratio of all central SRs is $0.0179 \pm 0.006$ ), this difference is significant. Correa \& Schaye (2020) found that at fixed halo mass in EAGLE, higher stellar mass galaxies formed in haloes that assembled earlier than lower stellar mass galaxies. Galaxies in haloes that assemble earlier also tend to have higher $\mathrm{BH}$-to-stellar mass ratios, indicating that AGN feedback can be more effective there (Bower et al. 2017). Since prolates tend to prefer gas-poor galaxy mergers (Figs 17 and 18), the more efficient AGN feedback can help promote their formation.

In Section 3.2 we showed the connection between environment and kinematic transformation in satellite galaxies that end up as SRs, as well as the connection between AGN feedback and quenching of central SRs. Here, we explore the connection between the kinematic class of satellites and centrals with their environment and stellar mass. Because our sample of SRs is small, we only split the subsample of satellites in two halo masses (left-hand panel of Fig. 20). We split
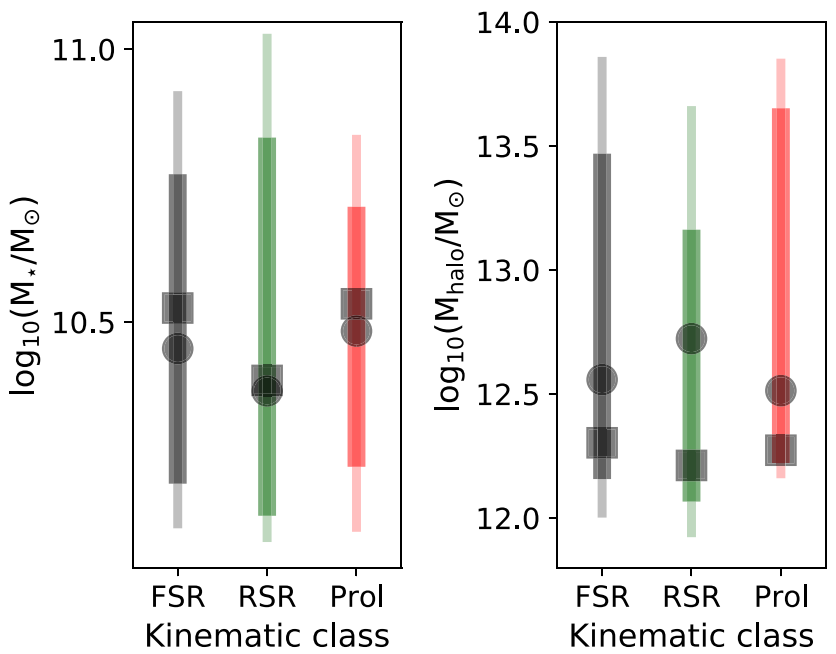

Figure 19. Median (filled circles) stellar (left-hand panel) and host halo (right-hand panel) mass of the SRs in the kinematic classes labelled in the $\mathrm{x}$-axis. The error bars show the 25th-75th (thicker lines) and 16th-84th (thinner lines) percentile ranges. The squares show the median of the subsample of central SRs in each kinematic class.

SR satellites by their host halo mass, below and above the median host halo mass, $\approx 10^{13.6} \mathrm{M}_{\odot}$. The median stellar masses of these two samples of satellites are very similar $\left(\approx 2.4 \times 10^{10} \mathrm{M}_{\odot}\right.$ and $\approx 2.7 \times 10^{10} \mathrm{M}_{\odot}$, respectively), hence yielding no bias in stellar mass when splitting by halo mass. We find an environmental trend, with SR satellites in low-mass haloes showing a preference for being FSRs, while those in high-mass haloes have a preference for being RSRs. Although prolate are found in both halo mass samples, their frequency is higher in high-mass haloes. Given that we find environment has a differential effect on $\lambda_{\mathrm{r}_{50} \text {,edge-on }}$ depending on the type of merger suffered by the SR prior to being accreted, it is likely that the environmental trend of Fig. 20 is at least in part driven by progenitor bias. To asses this, we study the incidence of different types of mergers between the satellite SRs in haloes of mass above and below $\approx 10^{13.6} \mathrm{M}_{\odot}$ and find that satellites hosted in haloes of mass $M_{\text {halo }} \lesssim 10^{13.6} \mathrm{M}_{\odot}$ are more (less) likely to have had major (minor) mergers compared to those hosted in more massive haloes, $M_{\text {halo }} \gtrsim 10^{13.6} \mathrm{M}_{\odot}$. The relative frequency of major and minor mergers in satellite SRs below and above the 

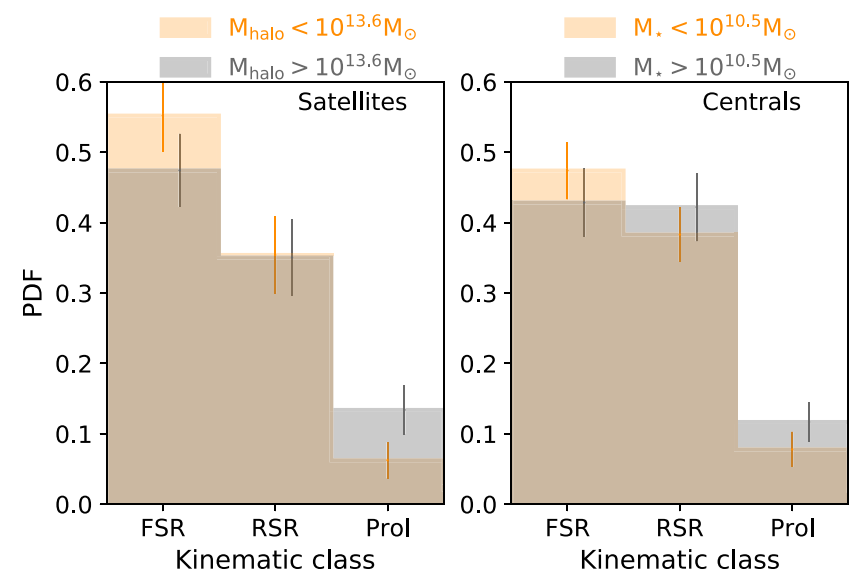

Figure 20. PDF of the kinematic classes of $z=0$ SRs separating satellite (left-hand panel) and central (right-hand panel) galaxies. For satellites, we show separately the distribution for galaxies above and below the median hot halo mass, $10^{13.6} \mathrm{M}_{\odot}$. For centrals, we separate them by their stellar mass, above and below the median, $10^{10.5} \mathrm{M}_{\odot}$, as labelled (the darker region is where histograms overlap). Error bars were computed from jackknife resampling and are displaced from the centre of the bin by arbitrary amounts to aid their visualization.

median host halo mass is $47 \pm 3$ per cent versus $37 \pm 2.8$ percent (major mergers) and $24 \pm 2$ per cent versus $32 \pm 3$ per cent (minor mergers), respectively, showing a significant effect of progenitor bias (uncertainties in percentages were computed from jackknife resampling). As Fig. 17 shows, FSRs are preferentially associated with major mergers, hence explaining why satellite FSRs are more common at $M_{\text {halo }} \lesssim 10^{13.6} \mathrm{M}_{\odot}$. We find no difference between the fraction of SRs that have exclusively very minor mergers or no mergers for satellites in haloes below/above $\approx 10^{13.6} \mathrm{M}_{\odot}$. The persistence of major mergers in satellites hosted by haloes of low masses partially explains the finding in Davison et al. (2020) of the ex-situ stellar fraction being higher in satellites at lower halo masses in EAGLE. Related to the difference between prolate satellite galaxies above/below $\approx 10^{13.6} \mathrm{M}_{\odot}$, the likely cause is the fact that galaxies tend to be gas-richer in lower mass haloes, and hence less likely to lead to a prolate galaxy, give the trends of Fig. 17.

The right-hand panel of Fig. 20 focuses now on central SRs, showing the distribution of kinematic classes in two bins of stellar mass, above/below the median of the sample, $\approx 10^{10.5} \mathrm{M}_{\odot}$. We see that massive central SRs have a preference for being FSRs, while at lower mass, FSRs and RSRs are similarly common. Prolate centrals tend to be similarly common below/above a stellar mass of $\approx 10^{10.5} \mathrm{M}_{\odot}$. In EAGLE, the fraction of galaxies that by $z=0$ have experienced a galaxy merger increases with stellar mass (Lagos et al. 2018 b), and the bottom panel of Fig. 6 shows that in particular the incidence of major mergers increases with stellar mass. Given the preference for FSRs to be associated with galaxy mergers with higher stellar mass ratios compared to RSRs (Fig. 17) it is not surprising that the massive central SRs have a preference for being FSRs.

\section{DISCUSSION AND CONCLUSIONS}

The possible formation paths of fast and slow rotators has been an area of intense research since the advent of IFS surveys, sampling hundreds of galaxies, revealed the existence of these populations (Emsellem et al. 2007, 2011). Their connection to galaxy mergers has been explored in hydrodynamical simulations, which have found varied and often contradictory results in the types of mergers that lead to the formation of SRs (Naab et al. 2014; Choi \& Yi 2017; Penoyre et al. 2017; Lagos et al. 2018a; Schulze et al. 2018). Generally to distinguish these two populations of rotators, both observations and simulations have generally employed parametric selections in the $\lambda_{\mathrm{r}}-\epsilon$ plane, motivated by the results of the ATLAS ${ }^{3 \mathrm{D}}$ survey (see Cappellari 2016 for a review). However, a significant problem that has become more evident is that these parametric selections lead to significant contamination (i.e. a high ratio of visual rotators to non-rotators) in the retrieved samples of SRs (van de Sande et al. 2021). This hinders the study of the formation paths of SRs in both observations and simulations, and could in part be responsible for the difficulty in isolating the main formation paths of SRs experienced in the latter.

To remedy this low SRs purity, here we employ visual classification of the stellar kinematic maps of galaxies in the EAGLE hydrodynamical simulations suite. We focused on galaxies with $M_{\star} \geq 10^{10} \mathrm{M}_{\odot}$ which have well-resolved kinematics (Lagos et al. 2017, 2018a). We place our galaxies in the context of the SAMI survey by creating kinematic maps using the SAMI specifications, which are readily available in the SIMSPIN package (see Section 2.2). We had five classifiers separating galaxies into six kinematic classes: flat SRs, round SRs, $2 \sigma$, prolates, unclear, and rotators. We found that $\geq 60$ per cent agreement among classifiers is reached in 90 percent of the classified galaxies, showing that the vast majority of galaxies can be cleanly separated into these kinematic classes. We use this sample to select unambiguous SRs in EAGLE, which correspond to flat or round SRs and prolate galaxies. Using the unambiguous SRs in EAGLE, we find that parametric classifications have at best a purity of 65 percent (i.e. 65 per cent of the galaxies that comply with the parametric selection of SRs are not considered as such by our visual classification), showing the requirement of the visual classification to isolate unambiguous SRs.

For the sample of unambiguous SRs in EAGLE, we study the connection to galaxy mergers, differences in intrinsic galaxy properties and the connection between quenching and kinematic transformation. We summarize our findings below:

(i) SRs with $M_{\star} \gtrsim 10^{10.8} \mathrm{M}_{\odot}$ have triaxiality, $T$, consistent with being prolates, $T>0.7$, while lower mass SRs span the whole range of $T$. Major and minor mergers lead to triaxial or prolate SRs $(T \gtrsim 0.5)$, while exclusively very minor mergers are largely associated with triaxial systems $(0.3 \lesssim T \lesssim 0.7)$. SRs that formed in the absence of mergers are oblate $(T \lesssim 0.2)$. These classes of SRs are clearly linked to different ex-situ stellar fractions, with SRs that had minor/major mergers or exclusively very minor mergers typically having $f_{\text {ex-situ }} \gtrsim$ 0.4 and $f_{\text {ex-situ }} \gtrsim 0.1$, respectively. SRs in the 'no merger' category have $f_{\text {ex-situ }} \lesssim 0.05$ (Fig. 7). This clearly shows that there is a class of SRs that forms in the absence of mergers in simulations (see also Choi \& Yi 2017). A higher fraction of galaxies in this class are satellites compared to SRs associated with galaxy mergers (Fig. 10).

(ii) SRs in the 'no merger' class tend to be more compact and have lower BH-to-stellar mass ratios than other SRs at fixed stellar mass (Fig. 7). They also tend to quench later (starting to drop below the main sequence at a look-back time $\approx 4.5 \mathrm{Gyr}$, compared to $\gtrsim 6 \mathrm{Gyr}$ for other SRs; Fig. 9). This leaves imprints on their stellar populations, with the 'no merger' SRs having lower $\alpha / \mathrm{Fe}$ (even below solar; Fig. 13) and higher Fe/H ratios than other SRs (Fig. 14).

(iii) We find that in most SRs quenching happens before kinematic transformation by $\approx 2$ Gyr (Figs 8 and 9). Most SRs quenched due to AGN feedback as evidenced by their overly massive BHs at the time they left the main sequence of SF (Fig. 11). These SRs tend to 
have quenching time-scales between 1.5 and $2.5 \mathrm{Gyr}$. The exception are satellite SRs that have not had mergers, which are quenched by the effect of environment. In the latter, quenching and kinematic transformation appear to happen in tandem (to within $0.2 \mathrm{Gyr}$ ), with the likely mechanism of kinematic transformation being interactions with the tidal field of the halo and central galaxy. The emerging picture is that in most SRs, quenching is required for galaxy mergers to more effectively decrease $\lambda_{\mathrm{r}}$.

(iv) We find that $\approx 50$ per cent of $z=0$ satellite SRs experienced satellite-satellite mergers, which were largely responsible for their SR fate (for reference only 20 percent of the general satellite population with $M_{\star} \geq 10^{10}$ experience satellite-satellite mergers). When focusing solely on $z=0$ satellite SRs that have not had satellite-satellite mergers, we find that environment was clearly responsible for the kinematic transformation of the subsamples of 'no merger' or 'exclusively very minor mergers' satellite SRs (Fig. 12). Nevertheless, we see an important effect of progenitor bias, with $\lambda_{\mathrm{r}}$ of the progenitors of $z=0$ satellite SRs that formed in the absence of mergers being higher than those that had mergers, regardless of whether these happen prior or after becoming satellites.

(v) Flat SRs are overly represented in the sample of SRs that had major mergers, while round SRs tend to prefer galaxies that had exclusively minor or very minor mergers (Figs 15 and 16). Prolate galaxies are predominantly connected to gas-poor galaxy mergers (Fig. 17). In the sample of flat and round SRs, we find that major and minor mergers associated with their formation tend to be gas-rich and gas-poor, respectively (Fig. 18).

(vi) Flat SRs tend to be more common in satellites hosted by massive haloes $\left(>10^{13.6} \mathrm{M}_{\odot}\right)$ and centrals of high stellar mass ( $>10^{10.5} \mathrm{M}_{\odot}$ ) due to the higher incidence of major mergers in these populations (Fig. 20). Prolates are also more common in these populations due to the higher incidence of gas-poor mergers. Prolate centrals have the highest stellar-to-halo mass ratios of all the SRs (Fig. 19), which we connect to those haloes preferentially forming earlier and having more AGN activity, as indicated in Correa \& Schaye (2020).

Although we find several trends between different types of mergers and SR's kinematic classes, we could not identify a single galaxy feature that can unambiguously indicate a given assembly history. This may not be surprising given the complexity of galaxy formation and the many physical processes that simultaneously take place. However, it does mean that the trends exposed here cannot be applied on a single galaxy basis.

There are some important limitations of our study. By visually inspecting many kinematic maps of EAGLE galaxies, we found a common feature of the stellar $\sigma$ being smaller at the centre and increasing towards the outskirts in $\approx 50$ percent of galaxies with $M_{\star}>10^{10} \mathrm{M}_{\odot}$ (see the top three panels of Fig. 1 for examples). The latter was found to be related to inverted stellar age radial profiles (where the central parts of galaxies are younger than the outer parts). We concluded that although feedback in EAGLE is sufficient to lead to integrated galaxy properties that agree well with observations (such as colour distribution, stellar mass function, global metallicities and oxygen abundance profiles, etc.; e.g. S15, Trayford et al. 2015, 2016; Segers et al. 2016; De Rossi et al. 2017; Katsianis et al. 2017; Tissera et al. 2019; Wright et al. 2019; Collacchioni et al. 2020), the imprints it leaves on the internal kinematic properties of galaxies is not always realistic. This physical limitation of the simulation needs to be addressed in upcoming realizations. A second limitation is inherent to the cosmological volume of EAGLE. After visual classification to find the unambiguous SRs in EAGLE, we are left with 479 galaxies. Although this sample is sufficient to provide us with the trends presented here, we often had to resort to studying the effect of a single quantity (e.g. stellar or halo mass), without controlling for others, making it difficult to disentangle (in some cases) the primary drivers of the trends above. Larger cosmological volumes, but retaining the sub-kpc resolution are required to address this limitation.

From the simulation's perspective, the future is promising. The advent of large cosmological volumes ( $\gtrsim 300 \mathrm{cMpc}$ ) at high enough spatial resolution (sub-kpc) will open the way to much more thorough studies connecting SRs and their diverse kinematic classes to a large range of merger parameters (not only mass and gas ratio, but also orbital parameters) as well as stellar mass and environment. In addition, small volume, but much higher resolution simulations, as to resolve the cold interstellar medium, will allow a better understanding of the formation of thin, flat disc galaxies, as well as how instabilities and galaxy mergers can lead to the formation of early-type, fast rotator galaxies.

Observations also promise significant progress over the next years. The fact that the kinematic transformation experienced by SRs in EAGLE happens at look-back times $\approx 2-6$ Gyr implies that the upcoming MUSE survey Middle Ages Galaxy Properties with Integral Field Spectroscopy (MAGPI; Foster et al. 2021) is ideally placed to unveil these transformations. The connection to $z=0$ surveys, such as SAMI, MaNGA, and Hector (Bryant et al. 2016), will complete this picture. We expect that in the next 3-5 yr IFS surveys observations will be able to place stringent constraints on the epoch of kinematic transformation and the (lack of) connection to star formation quenching.

\section{ACKNOWLEDGEMENTS}

$\mathrm{CL}, \mathrm{KEH}$, and $\mathrm{CF}$ have received funding from the ARC Centre of Excellence for All Sky Astrophysics in 3 Dimensions (ASTRO 3D), through project number CE170100013. EE thanks ASTRO 3D support during his visit to Australia. CL and CF are the receipts of an Australian Research Council Discovery Project (DP210101945) funded by the Australian Government. CL also thanks the MERAC Foundation for a Postdoctoral Research Award. JvdS acknowledges support of an Australian Research Council Discovery Early Career Research Award (project number DE200100461) funded by the Australian Government. LC is the recipient of an Australian Research Council Future Fellowship (FT180100066) funded by the Australian Government. This work used the DiRAC Data Centric system at Durham University, operated by the Institute for Computational Cosmology on behalf of the STFC DiRAC HPC Facility (www.dirac.ac.uk). This equipment was funded by BIS National E-infrastructure capital grant ST/K00042X/1, STFC capital grant ST/H008519/1, and STFC DiRAC Operations grant ST/K003267/1, and Durham University. DiRAC is part of the National E-Infrastructure. We acknowledge the Virgo Consortium for making their simulation data available. The EAGLE simulations were performed using the DiRAC-2 facility at Durham, managed by the ICC, and the PRACE facility Curie based in France at TGCC, CEA, Bruyeres-le-Chatel.

\section{DATA AVAILABILITY}

The EAGLE simulations are publicly available; see McAlpine et al. (2015), The EAGLE team (2017) for how to access EAGLE data. 


\section{REFERENCES}

Asplund M., Grevesse N., Sauval A. J., 2005, in Barnes T. G. III, Bash F. N., eds, ASP Conf. Ser. Vol. 336, Cosmic Abundances as Records of Stellar Evolution and Nucleosynthesis. Astron. Soc. Pac., San Francisco, p. 25

Bahé Y. M., Schaye J., Crain R. A., McCarthy I. G., Bower R. G., Theuns T., McGee S. L., Trayford J. W., 2017, MNRAS, 464, 508

Barsanti S. et al., 2021, ApJ, 906, 100

Bernardi M., Domínguez Sánchez H., Brownstein J. R., Drory N., Sheth R. K., 2019, MNRAS, 489, 5633

Béthermin M. et al., 2015, A\&A, 573, A113

Bois M. et al., 2011, MNRAS, 416, 1654

Bower R. G., Schaye J., Frenk C. S., Theuns T., Schaller M., Crain R. A., McAlpine S., 2017, MNRAS, 465, 32

Brough S. et al., 2017, ApJ, 844, 59

Bruzual G., Charlot S., 2003, MNRAS, 344, 1000 (BC03)

Bryant J. J. et al., 2015, MNRAS, 447, 2857

Bryant J. J. et al., 2016, in Evans C. J., Simard L., Takami H., eds, Proc. SPIE Conf. Ser. Vol. 9908, Ground-based and Airborne Instrumentation for Astronomy VI. SPIE, Bellingham, p. 99081F

Bundy K. et al., 2015, ApJ, 798, 7

Cañas R., Elahi P. J., Welker C., Lagos C. D. P., Power C., Dubois Y., Pichon C., 2019, MNRAS, 482, 2039

Cappellari M., 2016, ARA\&A, 54, 597

Cappellari M. et al., 2007, MNRAS, 379, 418

Cappellari M. et al., 2011, MNRAS, 413, 813

Choi H., Yi S. K., 2017, ApJ, 837, 68

Cole J. et al., 2020, ApJ, 890, L25

Collacchioni F., Lagos C. D. P., Mitchell P. D., Schaye J., Wisnioski E., Cora S. A., Correa C. A., 2020, MNRAS, 495, 2827

Correa C. A., Schaye J., 2020, MNRAS, 499, 3578

Correa C. A., Schaye J., Trayford J. W., 2019, MNRAS, 484, 4401

Cortese L. et al., 2019, MNRAS, 485, 2656

Crain R. A. et al., 2015, MNRAS, 450, 1937 (C15)

Croom S. M. et al., 2012, MNRAS, 421, 872

Dalla Vecchia C., Schaye J., 2012, MNRAS, 426, 140

Davies L. J. M. et al., 2018, MNRAS, 480, 768

Davies L. J. M. et al., 2019, MNRAS, 483, 5444

Davison T. A., Norris M. A., Pfeffer J. L., Davies J. J., Crain R. A., 2020, MNRAS, 497, 81

De Rossi M. E., Bower R. G., Font A. S., Schaye J., Theuns T., 2017, MNRAS, 472, 3354

Deeley S. et al., 2017, MNRAS, 467, 3934

Di Matteo P., Jog C. J., Lehnert M. D., Combes F., Semelin B., 2009, A\&A, 501, L9

Dolag K., Borgani S., Murante G., Springel V., 2009, MNRAS, 399, 497

Dressler A., 1980, ApJ, 236, 351

Ebrová I., Łokas E. L., Eliášek J., 2021, A\&A, 647, A103

Emsellem E. et al., 2007, MNRAS, 379, 401

Emsellem E. et al., 2011, MNRAS, 414, 888

Falcón-Barroso J. et al., 2017, A\&A, 597, A48

Foster C. et al., 2017, MNRAS, 472, 966

Foster C. et al., 2021, Publ. Astron. Soc. Austr., 38, e031

Furlong M. et al., 2015, MNRAS, 450, 4486

Furlong M. et al., 2017, MNRAS, 465, 722

González Delgado R. M. et al., 2015, A\&A, 581, A103

Graham M. T. et al., 2018, MNRAS, 477, 4711

Graham M. T., Cappellari M., Bershady M. A., Drory N., 2019, preprint (arXiv:1910.05139)

Green A. W. et al., 2018, MNRAS, 475, 716

Greene J. E., Janish R., Ma C.-P., McConnell N. J., Blakeslee J. P., Thomas J., Murphy J. D., 2015, ApJ, 807, 11

Greene J. E. et al., 2017, ApJ, 851, L33

Harborne K. E., Power C., Robotham A. S. G., 2020a, Publ. Astron. Soc. Austr., 37, e016

Harborne K. E., van de Sande J., Cortese L., Power C., Robotham A. S. G., Lagos C. D. P., Croom S., 2020b, MNRAS, 497, 2018
Jesseit R., Cappellari M., Naab T., Emsellem E., Burkert A., 2009, MNRAS, 397,1202

Jiang L., Helly J. C., Cole S., Frenk C. S., 2014, MNRAS, 440, 2115

Karademir G. S., Remus R.-S., Burkert A., Dolag K., Hoffmann T. L., Moster B. P., Steinwandel U. P., Zhang J., 2019, MNRAS, 487, 318

Katsianis A. et al., 2017, MNRAS, 472, 919

Krajnović D., Emsellem E., den Brok M., Marino R. A., Schmidt K. B., Steinmetz M., Weilbacher P. M., 2018, MNRAS, 477, 5327

Krajnović D. et al., 2020, A\&A, 635, A129

Kuntschner H. et al., 2010, MNRAS, 408, 97

Lagos C. D. P. et al., 2015, MNRAS, 452, 3815

Lagos C. D. P., Theuns T., Stevens A. R. H., Cortese L., Padilla N. D., Davis T. A., Contreras S., Croton D., 2017, MNRAS, 464, 3850

Lagos C. D. P., Schaye J., Bahé Y., Van de Sande J., Kay S. T., Barnes D., Davis T. A., Dalla Vecchia C., 2018a, MNRAS, 476, 4327

Lagos C. D. P. et al., 2018b, MNRAS, 473, 4956

Lange R. et al., 2016, MNRAS, 462, 1470

Li H., Mao S., Cappellari M., Graham M. T., Emsellem E., Long R. J., 2018a, ApJ, 863, L19

Li H., Mao S., Emsellem E., Xu D., Springel V., Krajnović D., 2018b, MNRAS, 473, 1489

Ma C.-P., Greene J. E., McConnell N., Janish R., Blakeslee J. P., Thomas J., Murphy J. D., 2014, ApJ, 795, 158

McAlpine S. et al., 2016, Astron. Comput., 15, 72

Marasco A., Crain R. A., Schaye J., Bahé Y. M., van der Hulst T., Theuns T., Bower R. G., 2016, MNRAS, 461, 2630

Naab T. et al., 2014, MNRAS, 444, 3357

Peng Y.-j. et al., 2010, ApJ, 721, 193

Penoyre Z., Moster B. P., Sijacki D., Genel S., 2017, MNRAS, 468, 3883

Pillepich A. et al., 2018, MNRAS, 473, 4077

Planck Collaboration XVI, 2014, A\&A, 571, A16

Pulsoni C., Gerhard O., Arnaboldi M., Pillepich A., Nelson D., Hernquist L., Springel V., 2020, A\&A, 641, A60

Qu Y. et al., 2017, MNRAS, 464, 1659

Rahmati A., Pawlik A. H., Raicevic M., Schaye J., 2013, MNRAS, 430, 2427

Robotham A. S. G., Bellstedt S., Lagos C. D. P., Thorne J. E., Davies L. J., Driver S. P., Bravo M., 2020, MNRAS, 495, 905

Rosas-Guevara Y. M. et al., 2015, MNRAS, 454, 1038

Rosito M. S., Tissera P. B., Pedrosa S. E., Lagos C. D. P., 2019, A\&A, 629, L3

Sánchez S. F. et al., 2012, A\&A, 538, A8

Santucci G. et al., 2020, ApJ, 896, 75

Schaye J., Dalla Vecchia C., 2008, MNRAS, 383, 1210

Schaye J. et al., 2015, MNRAS, 446, 521 (S15)

Schulze F., Remus R.-S., Dolag K., Burkert A., Emsellem E., van de Ven G., 2018, MNRAS, 480, 4636

Schulze F., Remus R.-S., Dolag K., Bellstedt S., Burkert A., Forbes D. A., 2020, MNRAS, 493, 3778

Scott N. et al., 2018, MNRAS, 481, 2299

Segers M. C., Schaye J., Bower R. G., Crain R. A., Schaller M., Theuns T., 2016, MNRAS, 461, L102

Smethurst R. J. et al., 2018, MNRAS, 473, 2679

Sparre M., Springel V., 2016, MNRAS, 462, 2418

Springel V., White S. D. M., Tormen G., Kauffmann G., 2001, MNRAS, 328, 726

Tacchella S. et al., 2019, MNRAS, 487, 5416

The EAGLE team, 2017, preprint (arXiv:1706.09899)

Thob A. C. R. et al., 2019, MNRAS, 485, 972

Tissera P. B., Rosas-Guevara Y., Bower R. G., Crain R. A., Lagos C. D. P., Schaller M., Schaye J., Theuns T., 2019, MNRAS, 482, 2208

Trayford J. W. et al., 2015, MNRAS, 452, 2879

Trayford J. W., Theuns T., Bower R. G., Crain R. A., Lagos C. D. P., Schaller M., Schaye J., 2016, MNRAS, 460, 3925

van de Sande J. et al., 2017a, MNRAS, 472, 1272

van de Sande J. et al., 2017b, ApJ, 835, 104 
van de Sande J. et al., 2019, MNRAS, 484, 869

van de Sande J. et al., 2021, MNRAS, 505, 3078

Veale M. et al., 2017, MNRAS, 464, 356

Walo-Martín D., Falcón-Barroso J., Dalla Vecchia C., Pérez I., Negri A., 2020, MNRAS, 494, 5652

Wang E. et al., 2018, ApJ, 860, 102

Wang B., Cappellari M., Peng Y., Graham M., 2020, MNRAS, 495, 1958

Weijmans A.-M. et al., 2014, MNRAS, 444, 3340
Wiersma R. P. C., Schaye J., Smith B. D., 2009a, MNRAS, 393, 99

Wiersma R. P. C., Schaye J., Theuns T., Dalla Vecchia C., Tornatore L., 2009b, MNRAS, 399, 574

Wright R. J., Lagos C. D. P., Davies L. J. M., Power C., Trayford J. W., Wong O. I., 2019, MNRAS, 487, 3740

This paper has been typeset from a $\mathrm{T}_{\mathrm{E}} \mathrm{X} / \mathrm{LAT}_{\mathrm{E}} \mathrm{X}$ file prepared by the author 